\title{
ERROR ESTIMATES FOR SEMILINEAR PARABOLIC CONTROL PROBLEMS IN THE ABSENCE OF TIKHONOV TERM*
}

\author{
EDUARDO CASAS ${ }^{\dagger}$, MARIANO MATEOS $^{\ddagger}$, AND ARND RÖSCH ${ }^{\S}$
}

\begin{abstract}
In this paper, we analyze optimal control problems of semilinear parabolic equations, where the controls are distributed and depend only on time. Box constraints for the controls are imposed and the cost functional does not involve the control itself, only the associated state. We prove second order optimality conditions for local strong minimizers, which are used to derive error estimates in the numerical approximation. First we estimate the difference between the discrete and continuous optimal states. In the last part, under an additional assumption on the optimal adjoint state, we prove error estimates for the controls and improve the estimates for the states.
\end{abstract}

Key words. optimal control, bang-bang control, semilinear parabolic equation, optimality conditions, error estimates

AMS subject classifications. 35K58, 49K20, 49M25

DOI. $10.1137 / 18 \mathrm{M} 117220 \mathrm{X}$

1. Introduction. In this paper, we consider a domain $\Omega \subset \mathbb{R}^{d}, d \leq 3$, with a Lipschitz boundary $\Gamma$. Given $T>0$ we denote $Q=\Omega \times(0, T)$ and $\Sigma=\Gamma \times(0, T)$. We will study the following control problem:

$$
\text { (P) } \min _{u \in U_{\text {ad }}} J(u),
$$

where

$$
U_{\text {ad }}=\left\{u \in L^{\infty}(0, T)^{m}: \alpha_{j} \leq u_{j}(t) \leq \beta_{j} \text { for a.a. } t \in(0, T), 1 \leq j \leq m\right\}
$$

with $-\infty<\alpha_{j}<\beta_{j}<+\infty$ for $1 \leq j \leq m, m \geq 1$ is a fixed integer number, and

$$
J(u)=\int_{Q} L\left(x, t, y_{u}(x, t)\right) d x d t .
$$

Above $y_{u}$ denotes the state associated to the control $u$ related by the following state equation:

$$
\left\{\begin{aligned}
\frac{\partial y_{u}}{\partial t}+A y_{u}+f\left(x, t, y_{u}\right) & =\sum_{j=1}^{m} u_{j}(t) g_{j}(x) \text { in } Q \\
y_{u} & =0 \text { on } \Sigma \\
y_{u}(0) & =y_{0} \text { in } \Omega .
\end{aligned}\right.
$$

On the data $A, f, g_{j}, y_{0}$, and $L$ we make the following assumptions:

\footnotetext{
${ }^{*}$ Received by the editors February 21, 2018; accepted for publication (in revised form) May 30, 2019; published electronically July 23, 2019.

https://doi.org/10.1137/18M117220X

Funding: The first two authors were partially supported by the Spanish Ministerio de Economía, Industria y Competitividad through research projects MTM2014-57531-P and MTM2017-83185-P.

†Departmento de Matemática Aplicada y Ciencias de la Computación, E.T.S.I. Industriales y de Telecomunicación, Universidad de Cantabria, 39005 Santander, Spain (eduardo.casas@unican.es).

‡Departamento de Matemáticas, Campus de Gijón, Universidad de Oviedo, 33203, Gijón, Spain (mmateos@uniovi.es).

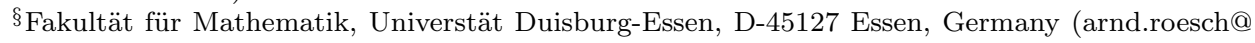
uni-due.de).
} 
(A1) $A$ denotes the elliptic operator

$$
A y=-\sum_{i, j=1}^{d} \partial_{x_{j}}\left(a_{i, j}(x) \partial_{x_{i}} y\right),
$$

where the coefficients $a_{i, j} \in L^{\infty}(\Omega)$ satisfy the uniform ellipticity condition

$$
\exists \lambda_{A}>0: \lambda_{A}|\xi|^{2} \leq \sum_{i, j=1}^{d} a_{i, j}(x) \xi_{i} \xi_{j} \forall \xi \in \mathbb{R}^{d} \text { and a.a. } x \in \Omega \text {. }
$$

(A2) We assume that $f: Q \times \mathbb{R} \rightarrow \mathbb{R}$ is a Carathéodory function of class $C^{2}$ with respect to the last variable satisfying the following properties:

$$
\exists C_{f} \in \mathbb{R}: \frac{\partial f}{\partial y}(x, t, y) \geq C_{f} \forall y \in \mathbb{R},
$$

$$
f(\cdot, \cdot, 0) \in L^{\hat{p}}\left(0, T ; L^{\hat{q}}(\Omega)\right) \text { for some } \hat{p}, \hat{q} \geq 2 \text { with } \frac{1}{\hat{p}}+\frac{d}{2 \hat{q}}<1,
$$

$$
\forall M>0 \exists C_{f, M}>0:\left|\frac{\partial^{j} f}{\partial y^{j}}(x, t, y)\right| \leq C_{f, M} \forall|y| \leq M \text { and } j=1,2,
$$

$\forall \rho>0$ and $\forall M>0 \exists \varepsilon>0$ such that

$$
\left|\frac{\partial^{2} f}{\partial y^{2}}\left(x, t, y_{1}\right)-\frac{\partial^{2} f}{\partial y^{2}}\left(x, t, y_{2}\right)\right|<\rho \forall\left|y_{1}\right|,\left|y_{2}\right| \leq M \text { with }\left|y_{1}-y_{2}\right|<\varepsilon
$$

for almost all $(x, t) \in Q$. Abusing notation, we will sometimes shorten $f(x, t, y)$ as $f(y), \frac{\partial f}{\partial y}(x, t, y)$ as $\frac{\partial f}{\partial y}(y)$ and $\frac{\partial^{2} f}{\partial y^{2}}(x, t, y)$ as $\frac{\partial^{2} f}{\partial y^{2}}(y)$ when this does not lead to confusion.

(A3) We assume that $\left\{g_{j}\right\}_{j=1}^{m} \subset L^{\infty}(\Omega)$ and there exist pairwise disjoint sets with positive Lebesgue measure $\left\{\omega_{j}\right\}_{j=1}^{m}$ such that $g_{j}$ vanishes outside $\omega_{j}$ and $g_{j}(x) \neq 0$ for a.a. $x \in \omega_{j}$.

(A4) For the initial datum we assume $y_{0} \in L^{\infty}(\Omega)$.

(A5) $L: Q \times \mathbb{R} \rightarrow \mathbb{R}$ is a Carathéodory function of class $C^{2}$ with respect to the last variable satisfying the following properties:

$L(\cdot, \cdot, 0) \in L^{1}(Q)$ and $\forall M>0 \exists \Psi_{M} \in L^{\hat{p}}\left(0, T ; L^{\hat{q}}(\Omega)\right)$ and $C_{L, M}$ such that

$$
\left|\frac{\partial L}{\partial y}(x, t, y)\right| \leq \Psi_{M}(x, t) \text { and }\left|\frac{\partial^{2} L}{\partial y^{2}}(x, t, y)\right| \leq C_{L, M} \forall|y| \leq M
$$

$$
\begin{aligned}
& \forall \rho>0 \text { and } \forall M>0 \exists \varepsilon>0 \text { such that } \\
& \left|\frac{\partial^{2} L}{\partial y^{2}}\left(x, t, y_{1}\right)-\frac{\partial^{2} L}{\partial y^{2}}\left(x, t, y_{2}\right)\right|<\rho \forall\left|y_{1}\right|,\left|y_{2}\right| \leq M \text { with }\left|y_{1}-y_{2}\right|<\varepsilon
\end{aligned}
$$

for almost all $(x, t) \in Q$.

Copyright ( by SIAM. Unauthorized reproduction of this article is prohibited. 
All these assumptions are required in the whole paper. Some additional assumptions will be specified later.

Though there are some recent papers concerning the second order optimality conditions for problems of type $(\mathrm{P})$, where the cost $J$ does not involve the control itself, this is still a field of active current research. There is vast literature on second order optimality conditions for control problems where the Tikhonov term appears in the functional $J$. However, the method of proof for these cases does not work for problems with an objective functional depending only on the state. In addition, the results for both type of problems are very different. The reader is referred to $[8,11,13,14,16,15,24]$ for sufficient second order conditions for bang-bang or bangsingular-bang control problems. Another issue that has gained the interest of the researchers is the derivation of second order conditions for strong local minimizers (Definition 3.1); see $[1,2,13,14]$. In this paper, we present a second order condition for strong local minimizers of $(\mathrm{P})$ based on the usual extended critical cone. This makes a difference with the only results in this direction proved in [11,13,14], where a different cone was required. The reader can find in section 3 a more detailed discussion on these issues.

A second novelty of this paper is the proof of error estimates for the difference between the discrete and continuous controls in the framework of parabolic control problems with bang-bang controls. As far as we know, the only results in this direction are obtained for linear state equations: in [17], a quadratic convergence order is obtained for the error with respect to the time step size in the case of bang-bang controls using a variational discretization; in [3], the authors study a time optimal control problem and obtain results similar to ours (compare [3, Table 1.1] and Theorem 6.5). For results in the case of the control of elliptic equations the reader can consult [18] for linear equations with variational discretization and [15] for semilinear equations with full discretization. In [15], a structural assumption on the optimal adjoint state is needed to prove the error estimates; see (6.1). In the absence of this condition, we still prove error estimates for the difference between the continuous and discrete optimal states; see also [9] for a similar result in the context of control of two dimensional evolutionary Navier-Stokes equations.

The plan of this paper is as follows. In section 2, we present some auxiliary results about the state equation and the differentiability of the cost functional. The first and second order optimality conditions are studied in section 3 . In section 4 we discretize the control problem and in section 5 we prove convergence of the discretizations and derive error estimates for the states. In section 6 we prove error estimates for the controls and improve the estimates for the states under the additional assumption (6.1). Finally, we present some numerical results in section 7 .

2. Auxiliary results. In this section we establish some properties of the state equation and the cost functional $J$. First, we state the existence and uniqueness and some regularity properties for the solution of (1.1).

TheOrem 2.1. For every $u \in L^{p}(0, T)^{m}$ with $p>1$ there exists a unique $y_{u} \in$ $Y:=L^{2}\left(0, T ; H_{0}^{1}(\Omega)\right) \cap L^{\infty}(Q)$ solution of (1.1). Moreover, there exists a constant $K_{p}>0$ independent of $u$ such that

$$
\begin{aligned}
\left\|y_{u}\right\|_{L^{2}\left(0, T ; H_{0}^{1}(\Omega)\right)}+ & \left\|y_{u}\right\|_{L^{\infty}(Q)} \\
& \leq K_{p}\left(\|u\|_{L^{p}(0, T)^{m}}+\left\|y_{0}\right\|_{L^{\infty}(\Omega)}+\|f(\cdot, \cdot, 0)\|_{L^{\hat{p}}\left(0, T ; L^{\hat{q}}(\Omega)\right)}\right) .
\end{aligned}
$$

Copyright (c) by SIAM. Unauthorized reproduction of this article is prohibited. 
Further, there exists a constant $M_{\infty}$ such that

$$
\left\|y_{u}\right\|_{L^{\infty}(Q)} \leq M_{\infty} \quad \forall u \in U_{\mathrm{ad}}
$$

This is a well known result. See, for instance, [7, Theorem 5.1] for some details concerning the nonlinearity of the equation. We also have the following continuity property.

LEMma 2.2. If $u_{k} \rightarrow u$ weakly in $L^{p}(0, T)^{m}$ for some $p>1$, then the strong convergence $\left\|y_{u_{k}}-y_{u}\right\|_{L^{\infty}(Q)} \rightarrow 0$ holds.

Proof. Define $z_{k}=y_{u_{k}}-y_{u}$. This function satisfies the equation

$$
\left\{\begin{aligned}
\frac{\partial z_{k}}{\partial t}+A z_{k}+f\left(y_{u_{k}}\right)-f\left(y_{u}\right) & =\sum_{j=1}^{m}\left(u_{k, j}(t)-u_{j}(t)\right) g_{j}(x) \text { in } Q \\
z_{k} & =0 \text { on } \Sigma \\
z_{k}(0) & =0 \text { in } \Omega .
\end{aligned}\right.
$$

By the mean value theorem, we know that there exist measurable functions $0<$ $\theta_{k}(x, t)<1$ such that, if we name $\hat{y}_{k}=y_{u}+\theta_{k}\left(y_{u_{k}}-y_{u}\right)$, then we have

$$
\left\{\begin{aligned}
\frac{\partial z_{k}}{\partial t}+A z_{k}+\frac{\partial f}{\partial y}\left(x, t, \hat{y}_{k}\right) z_{k} & =\sum_{j=1}^{m}\left(u_{k, j}(t)-u_{j}(t)\right) g_{j}(x) \text { in } Q \\
z_{k} & =0 \text { on } \Sigma \\
z_{k}(0) & =0 \text { in } \Omega .
\end{aligned}\right.
$$

Since the sequence $\left\{u_{k}\right\}_{k}$ converges weakly in $L^{p}(0, T)^{m}$, it is bounded in this space. Applying [20, Theorem III-10.1], we infer the existence of $\gamma$ and $C>0$ independent of $k$ such that

$$
\left\|z_{k}\right\|_{C^{\gamma, \frac{\gamma}{2}(\bar{Q})}} \leq C .
$$

Since $C^{\gamma, \frac{\gamma}{2}}(\bar{Q})$ is compactly embedded in $L^{\infty}(Q)$, we can extract a subsequence that converges in $L^{\infty}(Q)$ to some $z$. Taking the limit in (2.1), we deduce that $z=0$. Since all convergent subsequences of $\left\{z_{k}\right\}_{k}$ converge to 0 , the sequence itself converges to 0 .

Lemma 2.3. Given $u, v \in L^{p}(0, T)$ with $p>1$ and $u \neq v$, then $y_{u} \neq y_{v}$ holds.

Proof. Taking $z=y_{u}-y_{v}$, subtracting the equations satisfied by $y_{u}$ and $y_{v}$, and using the mean value theorem, we get for some $\hat{y}$ intermediate between $y_{u}$ and $y_{v}$

$$
\left\{\begin{aligned}
\frac{\partial z}{\partial t}+A z+\frac{\partial f}{\partial y}(x, t, \hat{y}) z & =\sum_{j=1}^{m}\left(u_{j}(t)-v_{j}(t)\right) g_{j}(x) \text { in } Q, \\
z & =0 \text { on } \Sigma \\
z(0) & =0 \text { in } \Omega .
\end{aligned}\right.
$$

From the assumption (A3), we deduce that the right-hand side of the above equation is not zero, and hence $z \neq 0$.

Given $p>1$, let us denote $G: L^{p}(0, T) \longrightarrow Y$ the mapping associating to each control the corresponding state $G(u)=y_{u}$.

Copyright $@$ by SIAM. Unauthorized reproduction of this article is prohibited. 
TheOREm 2.4. The control-to-state operator $G$ is of class $C^{2}$ and for every $u, v, w \in$ $L^{p}(0, T)^{m}, p>1$, we have that $z_{v}=G^{\prime}(u) v$ is the solution of

$$
\left\{\begin{aligned}
\frac{\partial z_{v}}{\partial t}+A z_{v}+\frac{\partial f}{\partial y}\left(x, t, y_{u}\right) z_{v} & =\sum_{j=1}^{m} v_{j}(t) g_{j}(x) \text { in } Q \\
z_{v} & =0 \text { on } \Sigma \\
z_{v}(0) & =0 \text { in } \Omega
\end{aligned}\right.
$$

and $z_{v, w}=G^{\prime \prime}(u)(v, w)$ solves the equation

$$
\left\{\begin{aligned}
\frac{\partial z_{v, w}}{\partial t}+A z_{v, w}+\frac{\partial f}{\partial y}\left(x, t, y_{u}\right) z_{v, w}+\frac{\partial^{2} f}{\partial y^{2}}\left(x, t, y_{u}\right) z_{v} z_{w} & =0 \text { in } Q \\
z_{v, w} & =0 \text { on } \Sigma \\
z_{v, w}(0) & =0 \text { in } \Omega .
\end{aligned}\right.
$$

We will use the following regularity result for linear equations.

Lemma 2.5. Consider $a_{0} \in L^{\infty}(Q)$ and $v \in L^{1}(0, T)^{m}$. Let $z \in L^{\infty}\left(0, T ; L^{2}(\Omega)\right) \cap$ $L^{2}\left(0, T ; H_{0}^{1}(\Omega)\right)$ be the solution of

$$
\left\{\begin{aligned}
\frac{\partial z}{\partial t}+A z+a_{0}(x, t) z & =\sum_{j=1}^{m} v_{j}(t) g_{j}(x) \text { in } Q \\
z & =0 \text { on } \Sigma \\
z(0) & =0 \text { in } \Omega .
\end{aligned}\right.
$$

Then, the following inequality holds:

$$
\|z\|_{L^{\infty}\left(0, T ; L^{2}(\Omega)\right)} \leq 2 \exp \left(\left\|a_{0}\right\|_{L^{\infty}(Q)} T\right) \max _{1 \leq j \leq m}\left\|g_{j}\right\|_{L^{2}(\Omega)}\|v\|_{L^{1}(0, T)^{m}} .
$$

Proof. By taking the change of variables $y(x, t)=\exp \left(-\left\|a_{0}\right\|_{L^{\infty}(Q)} t\right) z(x, t)$ the above equation is transformed into the following one:

$$
\left\{\begin{aligned}
\frac{\partial y}{\partial t}+A y+\left[a_{0}(x, t)+\left\|a_{0}\right\|_{L^{\infty}(Q)}\right] y & =\exp \left(-\left\|a_{0}\right\|_{L^{\infty}(Q)} t\right) \sum_{j=1}^{m} v_{j}(t) g_{j}(x) \text { in } Q \\
y & =0 \text { on } \Sigma \\
y(0) & =0 \text { in } \Omega
\end{aligned}\right.
$$

Multiplying the equation by $y$ and integrating in $\Omega \times(0, t)$ for $t \leq T$ we have

$$
\begin{aligned}
& \int_{0}^{t} \int_{\Omega} \frac{\partial y}{\partial t} y d x d s+\int_{0}^{t} \int_{\Omega}\left(A y+\left[a_{0}(x, s)+\left\|a_{0}\right\|_{L^{\infty}(Q)}\right] y\right) y d x d s \\
& \quad \leq \sum_{j=1}^{m} \int_{0}^{t} \int_{\omega_{j}} v_{j}(s) g_{j}(x) y(x, s) d x d s .
\end{aligned}
$$

Using the ellipticity condition (A1) and the fact that $\frac{\partial y}{\partial t} y=\frac{1}{2} \frac{\partial y^{2}}{\partial t}$ we get

$$
\|y(\cdot, t)\|_{L^{2}(\Omega)}^{2} \leq 2 \max _{1 \leq j \leq m}\left\|g_{j}\right\|_{L^{2}\left(\omega_{j}\right)}\|v\|_{L^{1}(0, T)^{m}}\|y\|_{L^{\infty}\left(0, T ; L^{2}(\Omega)\right)} \text { for a.a. } t \in[0, T] .
$$

We readily deduce that

$$
\|y\|_{L^{\infty}\left(0, T ; L^{2}(\Omega)\right)} \leq 2 \max _{1 \leq j \leq m}\left\|g_{j}\right\|_{L^{2}\left(\omega_{j}\right)}\|v\|_{L^{1}(0, T)^{m}}
$$

Inserting $z=\exp \left(\left\|a_{0}\right\|_{L^{\infty}(Q)} t\right) y(x, t)$ in this inequality, (2.4) follows.

Copyright (c) by SIAM. Unauthorized reproduction of this article is prohibited. 
Lemma 2.6. The solution operator $G$ is Lipschitz from $U_{\text {ad }}$ to $L^{\infty}\left(0, T ; L^{2}(\Omega)\right)$, i.e., there exists a constant $L_{1}>0$ such that

$$
\left\|y_{u_{1}}-y_{u_{2}}\right\|_{L^{\infty}\left(0, T ; L^{2}(\Omega)\right)} \leq L_{1}\left\|u_{1}-u_{2}\right\|_{L^{1}(0, T)^{m}} \quad \forall u_{1}, u_{2} \in U_{\mathrm{ad}} .
$$

Proof. Denote $z=y_{1}-y_{2}$. Using again the mean value theorem, there exists an intermediate $\hat{y}$ such that

$$
\left\{\begin{aligned}
\frac{\partial z}{\partial t}+A z+\frac{\partial f}{\partial y}(x, t, \hat{y}) z & =\sum_{j=1}^{m}\left(u_{1, j}(t)-u_{2, j}(t)\right) g_{j}(x) \text { in } Q, \\
z & =0 \text { on } \Sigma, \\
z(0) & =0 \text { in } \Omega .
\end{aligned}\right.
$$

Using Theorem 2.1, (1.4), and (2.4), we obtain the desired result.

Next, we state the differentiability properties of the objective functional. As usual, to every $u$, we relate the adjoint state $\varphi_{u}$ that satisfies

$$
\left\{\begin{aligned}
-\frac{\partial \varphi_{u}}{\partial t}+A^{*} \varphi_{u}+\frac{\partial f}{\partial y}\left(x, t, y_{u}\right) \varphi_{u} & =\frac{\partial L}{\partial y}\left(x, t, y_{u}\right) \text { in } Q, \\
\varphi_{u} & =0 \text { on } \Sigma, \\
\varphi_{u}(T) & =0 \text { in } \Omega,
\end{aligned}\right.
$$

where $A^{*}$ denotes the adjoint operator of $A$. Assumption (1.6) together with Theorem 2.1 implies that $\varphi_{u} \in L^{2}\left(0, T ; H_{0}^{1}(\Omega)\right) \cap C(\bar{Q})$ for every $u \in U_{\text {ad }}$

$$
\left\|\varphi_{u}\right\|_{L^{2}\left(0, T ; H_{0}^{1}(\Omega)\right)}+\left\|\varphi_{u}\right\|_{C(\bar{Q})} \leq K_{\infty} \quad \forall u \in U_{\mathrm{ad}}
$$

see [20, sections III-7 and III-10]. We also introduce the continuous functions

$$
\psi_{u, j}(t)=\int_{\omega_{j}} \varphi_{u}(x, t) g_{j}(x) d x, \quad 1 \leq j \leq m .
$$

The next theorem follows from the chain rule, Theorem 2.4, and assumptions (A2) and (A5).

Theorem 2.7. Given $p>1$, the functional $J: L^{p}(0, T)^{m} \longrightarrow \mathbb{R}$ is of class $C^{2}$ and for every $u, v, w \in L^{p}(0, T)^{m}$

$$
\begin{aligned}
J^{\prime}(u) v & =\sum_{j=1}^{m} \int_{0}^{T} \psi_{u, j}(t) v_{j}(t) d t \\
J^{\prime \prime}(u)(v, w) & =\int_{Q}\left(\frac{\partial^{2} L}{\partial y^{2}}\left(x, t, y_{u}\right)-\varphi_{u} \frac{\partial^{2} f}{\partial y^{2}}\left(x, t, y_{u}\right)\right) z_{v} z_{w} d x d t .
\end{aligned}
$$

Remark 2.8. The functionals $J^{\prime}(u)$ and $J^{\prime \prime}(u)$ can be extended to continuous linear and bilinear forms, respectively, in $L^{1}(0, T)^{m}$. Notice also that assumptions (A2) and (1.6), Theorem 2.1, Lemma 2.5, and (2.6) imply the existence of some $M_{2}>0$ such that

$$
J^{\prime \prime}(u)(v, w) \leq M_{2}\left\|z_{v}\right\|_{L^{2}(Q)}\left\|z_{w}\right\|_{L^{2}(Q)} \quad \forall u \in U_{\mathrm{ad}}, \forall v, w \in L^{1}(0, T)^{m} .
$$

Copyright (c) by SIAM. Unauthorized reproduction of this article is prohibited. 
3. First and second order optimality conditions. The existence of a global solution of $(\mathrm{P})$ follows in a standard way. Since $(\mathrm{P})$ is not a convex problem, we have to consider local solutions as well. Let us state precisely the different concepts of a local solution.

Definition 3.1. We say that $\bar{u}$ is an $L^{p}$-weak local minimum of $(\mathrm{P}), p \in[1,+\infty]$, if there exists some $\varepsilon>0$ such that

$$
J(\bar{u}) \leq J(u) \quad \forall u \in U_{\text {ad }} \text { with }\|\bar{u}-u\|_{L^{p}(0, T)^{m}} \leq \varepsilon .
$$

We say that $\bar{u}$ is a strong local minimum if there exists some $\varepsilon>0$ such that

$$
J(\bar{u}) \leq J(u) \quad \forall u \in U_{\text {ad }} \text { with }\left\|y_{\bar{u}}-y_{u}\right\|_{L^{\infty}(Q)} \leq \varepsilon .
$$

We say that $\bar{u}$ is a strict (weak or strong) local minimum if the above inequalities are strict for $u \neq \bar{u}$.

Lemma 3.2. The following properties hold:

1. $\bar{u}$ is an $L^{1}$-weak local minimum if and only if it is an $L^{p}$-weak local minimum for every $p \in[1,+\infty)$.

2. If $\bar{u}$ is an $L^{p}$-weak local minimum for some $p<+\infty$, then it is an $L^{\infty}$-weak local minimum.

3. If $\bar{u}$ is a strong local minimum, then it is an $L^{p}$-weak local minimum for all $p \in[1, \infty]$.

Proof. Statement 1 follows from the boundness of $U_{\text {ad }}$ in $L^{\infty}(0, T)^{m}$. Statement 2 follows from the inclusion $L^{\infty}(0, T)^{m} \subset L^{p}(0, T)^{m}$; cf. [10, p. 14].

To prove statement 3 , notice that the set $\left\{u \in U_{\text {ad }}:\|u-\bar{u}\|_{L^{1}(0, T)^{m}} \leq \delta\right\}$ is a subset of $\left\{u \in U_{\text {ad }}:\left\|\bar{y}-y_{u}\right\|_{L^{\infty}(Q)} \leq \rho\right\}$ if $\delta$ is sufficiently small.

First order optimality conditions read as follows.

THEOREM 3.3. Suppose $\bar{u}$ is a local solution of $(\mathrm{P})$ in any of the senses given in Definition 3.1. Then, there exist $\bar{y}$ and $\bar{\varphi}$ in $L^{2}\left(0, T ; H_{0}^{1}(\Omega)\right) \cap L^{\infty}(Q)$ such that

$$
\begin{aligned}
& \left\{\begin{aligned}
\frac{\partial \bar{y}}{\partial t}+A \bar{y}+f(x, t, \bar{y}) & =\sum_{j=1}^{m} \bar{u}_{j}(t) g_{j}(x) \text { in } Q, \\
\bar{y} & =0 \text { on } \Sigma, \\
\bar{y}(0) & =y_{0} \text { in } \Omega,
\end{aligned}\right. \\
& \left\{\begin{aligned}
-\frac{\partial \bar{\varphi}}{\partial t}+A^{*} \bar{\varphi}+\frac{\partial f}{\partial y}(x, t, \bar{y}) \bar{\varphi} & =\frac{\partial L}{\partial y}(x, t, \bar{y}) \text { in } Q, \\
\bar{\varphi} & =0 \text { on } \Sigma, \\
\bar{\varphi}(T) & =0 \text { in } \Omega,
\end{aligned}\right. \\
& \int_{0}^{T} \bar{\psi}_{j}(t)\left(u(t)-\bar{u}_{j}(t)\right) d t \geq 0 \forall \alpha_{j} \leq u(t) \leq \beta_{j} \text { a.e. in }(0, T), 1 \leq j \leq m,
\end{aligned}
$$

where

$$
\bar{\psi}_{j}(t)=\int_{\omega_{j}} g_{j}(x) \bar{\varphi}(x, t) d x .
$$

As usual, we can deduce from (3.1c) that

$$
\bar{u}_{j}(t)=\left\{\begin{array} { l l } 
{ \alpha _ { j } } & { \text { if } \overline { \psi } _ { j } ( t ) > 0 , } \\
{ \beta _ { j } } & { \text { if } \overline { \psi } _ { j } ( t ) < 0 }
\end{array} \text { and } \overline { \psi } _ { j } ( t ) \left\{\begin{array}{ll}
\geq 0 & \text { if } \bar{u}_{j}(t)=\alpha_{j}, \\
\leq 0 & \text { if } \bar{u}_{j}(t)=\beta_{j}, \\
=0 & \text { if } \alpha_{j}<\bar{u}_{j}(t)<\beta_{j} .
\end{array}\right.\right.
$$

Copyright (c) by SIAM. Unauthorized reproduction of this article is prohibited. 
Now, we establish the second order optimality conditions. In what follows, $\bar{u}$ will denote a control of $U_{\text {ad }}$ satisfying (3.1a)-(3.1c) along with the associated state $\bar{y}$ and adjoint state $\bar{\varphi}$. We define the cones of critical directions, for any $\tau \geq 0$,

$$
C_{\bar{u}}^{\tau}=\left\{v \in L^{2}(0, T)^{m}: v_{j}(t)\left\{\begin{array}{ll}
\geq 0 & \text { if } \bar{u}_{j}(t)=\alpha_{j} \\
\leq 0 & \text { if } \bar{u}_{j}(t)=\beta_{j} \\
=0 & \text { if }\left|\bar{\psi}_{j}(t)\right|>\tau
\end{array}\right\} .\right.
$$

For $\tau>0, C_{\bar{u}}^{\tau}$ can be considered as an extension of the classical cone of critical directions $C_{\bar{u}}=C_{\bar{u}}^{0}$. It is well known that $J^{\prime \prime}(\bar{u}) v^{2} \geq 0$ for all $v \in C_{\bar{u}}$ is a necessary second order condition for local optimality of $\bar{u}$; see, for instance, [5, section 3.2] or [12]. In order to have a minimal gap between the necessary and sufficient second order conditions, we would like to formulate a sufficient condition as $J^{\prime \prime}(\bar{u}) v^{2}>0$ for all $v \in C_{\bar{u}} \backslash\{0\}$ or $J^{\prime \prime}(\bar{u}) v^{2} \geq \delta\|v\|_{L^{2}(0, T)^{m}}^{2}$ for all $v \in C_{\bar{u}}$. Indeed, these two conditions are equivalent and sufficient if the Tikhonov term is included in the cost functional; see $[4,12]$ or [14]. However, when the Tikhonov term is absent, as in our case, these two conditions are no longer equivalent and they are not sufficient, in general, for local optimality. Furthermore, observe that $C_{\bar{u}}=\{0\}$ if $\bar{u}$ is a bang-bang control, and hence the above inequalities do not provide any information. Due to these arguments, some researchers have suggested considering extended cones as $C_{\bar{u}}^{\tau}$ with $\tau>0$; see [19] and [21]. Then the reader can be tempted to write the sufficient second order condition in this way: $J^{\prime \prime}(\bar{u}) v^{2} \geq \delta\|v\|_{L^{2}(0, T)^{m}}^{2}$ for all $v \in C_{\bar{u}}^{\tau}$. Unfortunately, this condition does not hold; see [8]. In [8] and [14], a different condition was assumed:

$$
\exists \delta>0 \text { and } \exists \tau>0: J^{\prime \prime}(\bar{u}) v^{2} \geq \delta\left\|z_{v}\right\|_{L^{2}(Q)}^{2} \quad \forall v \in C_{\bar{u}}^{\tau},
$$

where $z_{v}=G^{\prime}(\bar{u}) v$ is the solution of $(2.2)$ for $y_{u}=\bar{y}$. Then, it was proved that if $(\bar{u}, \bar{y}, \bar{\varphi})$ satisfy (3.1a)-(3.1c) and (3.3), then there exist $\varepsilon>0$ and $\kappa>0$ such that

$$
J(\bar{u})+\frac{\kappa}{2}\left\|y_{u}-\bar{y}\right\|_{L^{2}(Q)}^{2} \leq J(u) \forall u \in U_{\mathrm{ad}}:\|u-\bar{u}\|_{L^{2}(0, T)^{m}}<\varepsilon .
$$

This inequality proves that $\bar{u}$ is a strict $L^{2}$-weak local minimum of $(\mathrm{P})$. To deduce error estimates for finite element approximations for the state variable (see section 4 below) we would like to use (3.4) with $u=\bar{u}_{\sigma}$, the solution of the discrete problem. Nevertheless, the technique would work only if we have that $\bar{u}_{\sigma}$ converges strongly in $L^{2}(0, T)^{m}$ to $\bar{u}$, which is something we cannot deduce due to the absence of the Tikhonov regularization term. Define

A possible solution, also suggested in [14, Corollary 4], is to use a different cone.

$$
E_{\bar{u}}^{\tau}=\left\{v \in L^{2}(0, T)^{m}: v_{j}(t)\left\{\begin{array}{ll}
\geq 0 & \text { if } \bar{u}_{j}(t)=\alpha_{j}, \\
\leq 0 & \text { if } \bar{u}_{j}(t)=\beta_{j}
\end{array} \quad \text { and } J^{\prime}(\bar{u}) v \leq \tau\left\|z_{v}\right\|_{L^{2}(Q)}\right\} .\right.
$$

It is established in [14, Corollary 4] that if $(\bar{u}, \bar{y}, \bar{\varphi})$ satisfy (3.1a)-(3.1c) and

$$
\exists \tau>0 \text { and } \exists \delta>0: J^{\prime \prime}(\bar{u}) v^{2} \geq \delta\left\|z_{v}\right\|_{L^{2}(Q)}^{2} \forall v \in E_{\bar{u}}^{\tau},
$$

then there exist $\varepsilon>0$ and $\kappa>0$ such that

$$
J(\bar{u})+\frac{\kappa}{2}\left\|y_{u}-\bar{y}\right\|_{L^{2}(Q)}^{2} \leq J(u) \forall u \in U_{\mathrm{ad}}:\left\|y_{u}-\bar{y}\right\|_{L^{\infty}(Q)}<\varepsilon .
$$


Hence, $\bar{u}$ is a strict strong local minimum of $(\mathrm{P})$. The reader should observe that the cones $C_{\bar{u}}^{\tau}$ and $E_{\bar{u}}^{\tau}$ represent two different ways of extending $C_{\bar{u}}$; none of them is included in the other. We finish this section proving that condition (3.3) is also sufficient to deduce (3.6).

THEOREM 3.4. If $(\bar{u}, \bar{y}, \bar{\varphi})$ satisfy (3.1a)-(3.1c) and (3.3), then there exist $\varepsilon>0$ and $\kappa>0$ such that (3.6) holds.

Before proving this theorem, let us establish the following auxiliary lemma.

LEMMA 3.5. The following statements hold. then

1. for all $\gamma>0$ there exists $\varepsilon>0$ such that if $u \in U_{\mathrm{ad}}$ and $\left\|y_{u}-\bar{y}\right\|_{L^{\infty}(Q)}<\varepsilon$,

$$
\left\|y_{\bar{u}+\theta(u-\bar{u})}-\bar{y}\right\|<\gamma \forall \theta \in[0,1] .
$$
then

2. for all $\rho>0$ there exists $\varepsilon_{\rho}>0$ such that if $u \in U_{\text {ad }}$ and $\left\|y_{u}-\bar{y}\right\|_{L^{\infty}(Q)}<\varepsilon_{\rho}$,

$$
\left|\left[J^{\prime \prime}(\bar{u}+\theta(u-\bar{u}))-J^{\prime \prime}(\bar{u})\right] v^{2}\right| \leq \rho\left\|z_{v}\right\|_{L^{2}(Q)}^{2} \forall v \in L^{2}(0, T)^{m} \forall \theta \in[0,1] .
$$

Proof. According to [14, Lemma 6], for every $\rho>0$ there exists $\varepsilon_{\rho}>0$ such that if $u \in U_{\mathrm{ad}}$ and $\left\|y_{u}-\bar{y}\right\|_{L^{\infty}(Q)}<\varepsilon_{\rho}$, then

$$
\left|\left(J^{\prime \prime}(u)-J^{\prime \prime}(\bar{u})\right) v^{2}\right| \leq \rho\left\|z_{v}\right\|_{L^{2}(Q)}^{2} \forall v \in L^{2}(0, T)^{m} .
$$

Therefore, (3.8) is an immediate consequence of (3.7) and this inequality. Let us prove (3.7). Take $u \in U_{\text {ad }}$ such that $\left\|y_{u}-\bar{y}\right\|<\varepsilon$ with $\varepsilon>0$ to be defined later. Let us prove that (3.7) holds for an arbitrary $\theta \in(0,1)$. Consider $z=y_{\bar{u}+\theta(u-\bar{u})}-\left[\bar{y}+\theta\left(y_{u}-\bar{y}\right)\right]$. The function $z$ satisfies

$$
\left\{\begin{aligned}
\frac{\partial z}{\partial t}+A z+f\left(y_{\bar{u}+\theta(u-\bar{u})}\right)-\left[f(\bar{y})+\theta\left(f\left(y_{u}\right)-f(\bar{y})\right)\right] & =0 \text { in } Q \\
z & =0 \text { on } \Sigma \\
z(0) & =0 \text { in } \Omega .
\end{aligned}\right.
$$

Applying the mean value theorem, we have that there exist measurable functions $0<\theta_{1}(x, t)<1$ and $0<\theta_{2}(x, t)<1$ such that, if we name

$$
y_{1}=\bar{y}+\theta_{1}\left(y_{\bar{u}+\theta(u-\bar{u})}-\bar{y}\right) \text { and } y_{2}=\bar{y}+\theta_{2}\left(y_{u}-\bar{y}\right),
$$

we have

$$
\frac{\partial z}{\partial t}+A z+\frac{\partial f}{\partial y}\left(y_{1}\right)\left(y_{\bar{u}+\theta(u-\bar{u})}-\bar{y}\right)-\theta \frac{\partial f}{\partial y}\left(y_{2}\right)\left(y_{u}-\bar{y}\right)=0 \text { in } Q .
$$

Note that

$$
y_{\bar{u}+\theta(u-\bar{u})}-\bar{y}=z+\theta\left(y_{u}-\bar{y}\right) .
$$

So, applying again the mean value theorem, there exists another measurable function $0<\theta_{3}(x, t)<1$ such that, if we name $y_{3}=y_{2}+\theta_{3}\left(y_{1}-y_{2}\right)$, then

$$
\begin{aligned}
\frac{\partial f}{\partial y}\left(y_{1}\right)( & \left.y_{\bar{u}+\theta(u-\bar{u})}-\bar{y}\right)-\theta \frac{\partial f}{\partial y}\left(y_{2}\right)\left(y_{u}-\bar{y}\right) \\
= & \frac{\partial f}{\partial y}\left(y_{1}\right) z+\theta \frac{\partial f}{\partial y}\left(y_{1}\right)\left(y_{u}-\bar{y}\right)-\theta \frac{\partial f}{\partial y}\left(y_{2}\right)\left(y_{u}-\bar{y}\right) \\
= & \frac{\partial f}{\partial y}\left(y_{1}\right) z+\theta \frac{\partial^{2} f}{\partial y^{2}}\left(y_{3}\right)\left(y_{1}-y_{2}\right)\left(y_{u}-\bar{y}\right) .
\end{aligned}
$$

Copyright (c) by SIAM. Unauthorized reproduction of this article is prohibited. 
So the function $z$ satisfies

$$
\frac{\partial z}{\partial t}+A z+\frac{\partial f}{\partial y}\left(y_{1}\right) z=-\theta \frac{\partial^{2} f}{\partial y^{2}}\left(y_{3}\right)\left(y_{1}-y_{2}\right)\left(y_{u}-\bar{y}\right) \text { in } Q .
$$

From Theorem 2.1 it is clear that $y_{1}, y_{2}$, and $y_{3}$ are uniformly bounded in $L^{\infty}(Q)$ by $M_{\infty}$. Using assumption (A2) and the fact that $\left\|y_{u}-\bar{y}\right\|_{L^{\infty}(Q)}<\varepsilon$, we deduce with [20, section III.7] the existence of a constant $C_{1}>0$ independent of $u$ such that

$$
\|z\|_{L^{\infty}(Q)} \leq C_{1} \varepsilon .
$$

With (3.9), (3.10), $0<\theta<1$, and the condition $\left\|y_{u}-\bar{y}\right\|_{L^{\infty}(Q)}<\varepsilon$, we deduce that

$$
\left\|y_{\bar{u}+\theta(u-\bar{u})}-\bar{y}\right\|_{L^{\infty}(Q)}<\left(C_{1}+1\right) \varepsilon .
$$

So (3.7) holds if we define

$$
\varepsilon=\frac{\gamma}{1+C_{1}}
$$

Proof of Theorem 3.4. Consider $u \in U_{\text {ad }}$ such that $\left\|y_{u}-\bar{y}\right\|_{L^{\infty}(Q)}<\varepsilon$, where $\varepsilon$ will be fixed later independently of $u$; see (3.21) below. A second order Taylor expansion yields the existence of $\theta \in(0,1)$ such that

$$
J(u)=J(\bar{u})+J^{\prime}(\bar{u})(u-\bar{u})+\frac{1}{2} J^{\prime \prime}(\bar{u}+\theta(u-\bar{u}))(u-\bar{u})^{2} .
$$

Define for $1 \leq j \leq m$

$$
v_{j}(t)=\left\{\begin{array}{cc}
u_{j}(t)-\bar{u}_{j}(t) & \text { if }\left|\bar{\psi}_{j}(t)\right| \leq \tau \\
0 & \text { otherwise }
\end{array}\right.
$$

and $w=u-\bar{u}-v$. It is clear that $v \in C_{\bar{u}}^{\tau}$. Taking into account (3.2) we also have

$$
\begin{aligned}
J^{\prime}(\bar{u})(u-\bar{u}) & =\sum_{j=1}^{m} \int_{0}^{T} \bar{\psi}_{j}(t)\left(u_{j}(t)-\bar{u}_{j}(t)\right) d t \\
& \geq \sum_{j=1}^{m} \int_{0}^{T} \bar{\psi}_{j}(t) w_{j}(t) d t \geq \tau \sum_{j=1}^{m} \int_{0}^{T}\left|w_{j}(t)\right| d t=\tau\|w\|_{L^{1}(0, T)^{m}} .
\end{aligned}
$$

Using (3.12), $u-\bar{u}=v+w,(3.13), v \in C_{\bar{u}}^{\tau}$, the second order sufficient condition (3.3), the estimate (2.10) for the bilinear form $J^{\prime \prime}(\bar{u})$, and Young's inequality, we obtain

$$
\begin{aligned}
J(u) \geq & J(\bar{u})+\tau\|w\|_{L^{1}(0, T)^{m}}+\frac{1}{2} J^{\prime \prime}(\bar{u}) v^{2}+\frac{1}{2} J^{\prime \prime}(\bar{u}) w^{2}+J^{\prime \prime}(\bar{u})(v, w) \\
& +\frac{1}{2}\left[J^{\prime \prime}(\bar{u}+\theta(u-\bar{u}))-J^{\prime \prime}(\bar{u})\right](u-\bar{u})^{2} \\
\geq & J(\bar{u})+\tau\|w\|_{L^{1}(0, T)^{m}}+\frac{\delta}{2}\left\|z_{v}\right\|_{L^{2}(Q)}^{2}-\frac{M_{2}}{2}\left\|z_{w}\right\|_{L^{2}(Q)}^{2} \\
& -M_{2}\left\|z_{v}\right\|_{L^{2}(Q)}\left\|z_{w}\right\|_{L^{2}(Q)}+\frac{1}{2}\left[J^{\prime \prime}(\bar{u}+\theta(u-\bar{u}))-J^{\prime \prime}(\bar{u})\right](u-\bar{u})^{2} \\
\geq & J(\bar{u})+\tau\|w\|_{L^{1}(0, T)^{m}}+\frac{\delta}{4}\left\|z_{v}\right\|_{L^{2}(Q)}^{2}-M_{2}\left(\frac{1}{2}+\frac{M_{2}}{\delta}\right)\left\|z_{w}\right\|_{L^{2}(Q)}^{2} \\
& +\frac{1}{2}\left[J^{\prime \prime}(\bar{u}+\theta(u-\bar{u}))-J^{\prime \prime}(\bar{u})\right](u-\bar{u})^{2} .
\end{aligned}
$$

Copyright (c) by SIAM. Unauthorized reproduction of this article is prohibited. 
Using this inequality and Lemma 3.5 with $\rho=\delta / 8$ and $v=u-\bar{u}$ and assuming that $\varepsilon \leq \varepsilon_{\rho}$ given at the start of the proof, we have

$$
\begin{aligned}
J(u) \geq & J(\bar{u})+\tau\|w\|_{L^{1}(0, T)^{m}}+\frac{\delta}{4}\left\|z_{v}\right\|_{L^{2}(Q)}^{2}-M_{2}\left(\frac{1}{2}+\frac{M_{2}}{\delta}\right)\left\|z_{w}\right\|_{L^{2}(Q)}^{2} \\
& -\frac{\delta}{16}\left\|z_{u-\bar{u}}\right\|_{L^{2}(Q)^{2}}^{2} .
\end{aligned}
$$

We notice now that

$$
\begin{aligned}
\left\|z_{u-\bar{u}}\right\|_{L^{2}(Q)}^{2} & =\left\|z_{v+w}\right\|_{L^{2}(Q)}^{2} \leq\left\|z_{v}\right\|_{L^{2}(Q)}^{2}+\left\|z_{w}\right\|_{L^{2}(Q)}^{2}+2\left\|z_{v}\right\|_{L^{2}(Q)}\left\|z_{w}\right\|_{L^{2}(Q)} \\
& \leq 2\left\|z_{v}\right\|_{L^{2}(Q)}^{2}+2\left\|z_{w}\right\|_{L^{2}(Q)}^{2}
\end{aligned}
$$

and hence $\left\|z_{v}\right\|_{L^{2}(Q)}^{2} \geq \frac{1}{2}\left\|z_{u-\bar{u}}\right\|_{L^{2}(Q)}^{2}-\left\|z_{w}\right\|_{L^{2}(Q)}^{2}$. Inserting this in (3.15) we get

$$
J(u) \geq J(\bar{u})+\frac{\delta}{16}\left\|z_{u-\bar{u}}\right\|_{L^{2}(\Omega)}^{2}+\tau\|w\|_{L^{1}(0, T)^{m}}-M_{2}\left(\frac{1}{2}+\frac{M_{2}}{\delta}+\frac{\delta}{4}\right)\left\|z_{w}\right\|_{L^{2}(Q)}^{2} .
$$

Let us estimate $\tau\|w\|_{L^{1}(0, T)^{m}}$. Using again (3.13) and the expression for the derivative of $J$ obtained directly by the chain rule, we have

$$
\begin{aligned}
\tau\|w\|_{L^{1}(0, T)^{m}} & \leq J^{\prime}(\bar{u})(u-\bar{u})=\int_{Q} \frac{\partial L}{\partial y}(x, t, \bar{y}) z_{u-\bar{u}} d x d t \\
& \leq\left\|\frac{\partial L}{\partial y}(x, t, \bar{y})\right\|_{L^{2}(Q)}\left\|z_{u-\bar{u}}\right\|_{L^{2}(Q)} .
\end{aligned}
$$

Define now $\eta=y_{u}-\left(\bar{y}+z_{u-\bar{u}}\right)$. The function $\eta$ satisfies the equation

$$
\left\{\begin{aligned}
\frac{\partial \eta}{\partial t}+A \eta+f\left(y_{u}\right)-f(\bar{y})-\frac{\partial f}{\partial y}(\bar{y}) z_{u-\bar{u}} & =0 \text { in } Q \\
\eta & =0 \text { on } \Sigma \\
\eta(0) & =0 \text { in } \Omega .
\end{aligned}\right.
$$

Using a second order Taylor expansion and the definition of $\eta$, we have that there exists $0<\hat{\theta}(x, t)<1$ such that, if we name $\hat{y}=\bar{y}+\hat{\theta}\left(y_{u}-\bar{y}\right)$, we have that

$$
\frac{\partial \eta}{\partial t}+A \eta+\frac{\partial f}{\partial y}(\bar{y}) \eta=-\frac{1}{2} \frac{\partial^{2} f}{\partial y^{2}}(\hat{y})\left(y_{u}-\bar{y}\right)^{2} \text { in } Q .
$$

Again from assumption (A2), and the condition $\left\|y_{u}-\bar{y}\right\|_{L^{\infty}(Q)}<\varepsilon$, we deduce the existence of a constant $C_{1}>0$ independent of $\varepsilon$ and $u$ such that

$$
\|\eta\|_{L^{2}(Q)} \leq C_{1} \varepsilon\left\|y_{u}-\bar{y}\right\|_{L^{2}(Q)} \leq \sqrt{T|\Omega|} C_{1} \varepsilon^{2} .
$$

Using this, the definition of $\eta$, and $\left\|y_{u}-\bar{y}\right\|_{L^{\infty}(Q)}<\varepsilon$, we infer

$$
\left\|z_{u-\bar{u}}\right\|_{L^{2}(Q)} \leq\|\eta\|_{L^{2}(Q)}+\left\|y_{u}-\bar{y}\right\|_{L^{2}(Q)} \leq\left(C_{1} \varepsilon+1\right) \sqrt{T|\Omega|} \varepsilon .
$$

Now, from (3.17) we deduce the existence of a constant $C_{2}$ independent of $u$ such that

$$
\tau\|w\|_{L^{1}(0, T)^{m}} \leq C_{2} \varepsilon
$$

Copyright (c) by SIAM. Unauthorized reproduction of this article is prohibited. 
Next we use (2.4) and (3.19) to obtain

$$
\left\|z_{w}\right\|_{L^{2}(Q)}^{2} \leq T\left\|z_{w}\right\|_{L^{\infty}\left(0, T ; L^{2}(\Omega)\right)}^{2} \leq \frac{C_{3}}{\tau} \varepsilon\|w\|_{L^{1}(0, T)^{m}}
$$

and, hence,

$$
\tau\|w\|_{L^{1}(0, T)^{m}} \geq \frac{\tau^{2}}{C_{3} \varepsilon}\left\|z_{w}\right\|_{L^{2}(Q)}^{2} .
$$

Using this and (3.16), we have that

$$
J(u) \geq J(\bar{u})+\frac{\delta}{16}\left\|z_{u-\bar{u}}\right\|_{L^{2}(\Omega)}^{2}+\left[\frac{\tau^{2}}{C_{3} \varepsilon}-M_{2}\left(\frac{1}{2}+\frac{M_{2}}{\delta}+\frac{\delta}{4}\right)\right]\left\|z_{w}\right\|_{L^{2}(Q)}^{2} .
$$

So the condition

$$
\varepsilon \leq \min \left\{\varepsilon_{\rho}, \frac{\tau^{2}}{C_{3} M_{2}\left(\frac{1}{2}+\frac{M_{2}}{\delta}+\frac{\delta}{4}\right)}\right\}
$$

yields

$$
J(u) \geq J(\bar{u})+\frac{\delta}{16}\left\|z_{u-\bar{u}}\right\|_{L^{2}(\Omega)}^{2}
$$

Finally, using again the definition of the function $\eta$ and (3.18), we have that

$$
\left\|y_{u}-\bar{y}\right\|_{L^{2}(Q)} \leq\|\eta\|_{L^{2}(Q)}+\left\|z_{u-\bar{u}}\right\|_{L^{2}(Q)} \leq C_{1} \varepsilon\left\|y_{u}-\bar{y}\right\|_{L^{2}(Q)}+\left\|z_{u-\bar{u}}\right\|_{L^{2}(Q)} .
$$

So imposing also $\varepsilon<\frac{1}{2 C_{1}}$ we have that $\left\|z_{u-\bar{u}}\right\|_{L^{2}(Q)} \geq \frac{1}{2}\left\|y_{u}-\bar{y}\right\|_{L^{2}(Q)}$. Using this and (3.22) we obtain

$$
J(u) \geq J(\bar{u})+\frac{\delta}{64}\left\|y_{u}-\bar{y}\right\|_{L^{2}(\Omega)}^{2},
$$

and the result follows for $\kappa=\delta / 64$.

Remark 3.6. In the above proof, we have established that

$$
\begin{aligned}
J^{\prime}(\bar{u})(u-\bar{u}) & \geq \tau\|w\|_{L^{1}(0, T)^{m}}, \\
J^{\prime \prime}(\bar{u}+\theta(u-\bar{u}))(u-\bar{u})^{2} & \geq \frac{\delta}{8}\left\|z_{u-\bar{u}}\right\|_{L^{2}(Q)}^{2}-C\left\|z_{w}\right\|_{L^{2}(Q)}^{2} \forall \theta \in[0,1],
\end{aligned}
$$

assuming that $u \in U_{\text {ad }}$ and $\left\|y_{u}-\bar{y}\right\|_{L^{\infty}(Q)}<\varepsilon$ for any $0<\varepsilon \leq \varepsilon_{0}$ with $\varepsilon_{0}$ conveniently chosen. From these two inequalities, we get for any number $\rho>0$

$\rho J^{\prime}(\bar{u})(u-\bar{u})+J^{\prime \prime}(\bar{u}+\theta(u-\bar{u}))(u-\bar{u})^{2} \geq \rho \tau\|w\|_{L^{1}(0, T)^{m}}+\frac{\delta}{8}\left\|z_{u-\bar{u}}\right\|_{L^{2}(Q)}^{2}-C\left\|z_{w}\right\|_{L^{2}(Q)}^{2}$.

Now, using (3.20) and replacing the requirement (3.21) by $\varepsilon \leq \rho \tau^{2} /\left(C_{3} C\right)$ we get that

$$
\rho \tau\|w\|_{L^{1}(0, T)^{m}}-C\left\|z_{w}\right\|_{L^{2}(Q)}^{2} \geq 0 .
$$

Hence, we obtain that

$$
\rho J^{\prime}(\bar{u})(u-\bar{u})+J^{\prime \prime}(\bar{u}+\theta(u-\bar{u}))(u-\bar{u})^{2} \geq \frac{\delta}{8}\left\|z_{u-\bar{u}}\right\|_{L^{2}(Q)}^{2} .
$$

Finally, taking again $\varepsilon$ smaller if necessary, we get as in the above proof $\left\|z_{u-\bar{u}}\right\|_{L^{2}(Q)} \geq$ $\frac{1}{2}\left\|y_{u}-\bar{y}\right\|_{L^{2}(Q)}$, and thus there exist $\lambda>0$ and $\varepsilon_{\rho}>0$ such that for $\left\|y_{u}-\bar{y}\right\|_{L^{\infty}(Q)} \leq \varepsilon_{\rho}$

$$
\rho J^{\prime}(\bar{u})(u-\bar{u})+J^{\prime \prime}(\bar{u}+\theta(u-\bar{u}))(u-\bar{u})^{2} \geq \frac{\lambda}{2}\left\|y_{u}-\bar{y}\right\|_{L^{2}(Q)}^{2} .
$$

Copyright (c) by SIAM. Unauthorized reproduction of this article is prohibited. 
4. Numerical approximation of the control problem (P). The goal of this section is to get a discrete version of the control problem $(\mathrm{P})$. To this end, we need to make some additional hypotheses to (A1)-(A5) that we will assume in the rest of the paper.

(H1) $\Omega$ is a polygonal (if $d=2$ ) or polyhedral (if $d=3$ ) convex domain.

(H2) The initial state $y_{0}$ belongs to $H^{2}(\Omega) \cap H_{0}^{1}(\Omega)$, the coefficients $a_{i j}$ of $A$ are Lipschitz functions in $\bar{\Omega}$, and $f(\cdot, \cdot, 0) \in L^{\infty}(Q)$.

(H3) For every $M>0$ there exists a constant $C_{L, M}$ such that

$$
\left|\frac{\partial L}{\partial y}(x, t, y)\right| \leq C_{L, M} \quad \text { for a.a. }(x, t) \in Q \forall|y| \leq M .
$$

These assumptions imply extra regularity for the states $y_{u}$ and adjoint states $\varphi_{u}$.

TheOrem 4.1. For every $u \in U_{\text {ad }}$ we have that $y_{u}, \varphi_{u} \in W^{1, p}\left(0, T ; L^{2}(\Omega)\right) \cap$ $L^{p}\left(0, T ; H^{2}(\Omega)\right)$ for all $p<\infty$. Furthermore, there exists a constant $M_{0}$ independent of $u$ and $p$ such that

$$
\begin{aligned}
& \left\|\partial_{t} y_{u}\right\|_{L^{p}\left(0, T ; L^{2}(\Omega)\right)}+\left\|y_{u}\right\|_{L^{p}\left(0, T ; H^{2}(\Omega)\right)} \\
& \quad \leq M_{0} \frac{p^{2}}{p-1}\left(\|u\|_{L^{\infty}(0, T)^{m}}+\|f(\cdot, \cdot, 0)\|_{L^{\infty}\left(0, T ; L^{2}(\Omega)\right)}+\left\|y_{0}\right\|_{H^{2}(\Omega)}\right), \\
& \left\|\partial_{t} \varphi_{u}\right\|_{L^{p}\left(0, T ; L^{2}(\Omega)\right)}+\left\|\varphi_{u}\right\|_{L^{p}\left(0, T ; H^{2}(\Omega)\right)} \leq M_{0} \frac{p^{2}}{p-1} C_{L, M_{\infty}},
\end{aligned}
$$

where $M_{\infty}$ was introduced in Theorem 2.1 and $C_{L, M_{\infty}}$ is defined in assumption (H3).

The reader is referred to [22] for the proof.

We consider (cf. [6, Definition (4.4.13)]) a quasi-uniform family of triangulations $\left\{\mathcal{K}_{h}\right\}_{h>0}$ of $\bar{\Omega}$ and a quasi-uniform family of partitions of size $\tau$ of $[0, T], 0=t_{0}<$ $t_{1}<\cdots<t_{N_{\tau}}=T$. We will denote $N_{h}$ and $N_{I, h}$ the number of nodes and interior nodes of $\mathcal{K}_{h}, I_{k}=\left(t_{k-1}, t_{k}\right), \tau_{k}=t_{k}-t_{k-1}, \tau=\max \left\{\tau_{k}\right\}$, and $\sigma=(h, \tau)$. In what follows we will use the notation $|\sigma|=\tau+h$.

Now we consider the finite dimensional spaces

$$
\begin{aligned}
& Y_{h}=\left\{z_{h} \in C(\bar{\Omega}): z_{h \mid K} \in P_{1}(K) \forall K \in \mathcal{K}_{h} \text { and } z_{h} \equiv 0 \text { on } \Gamma\right\} \\
& \mathcal{Y}_{\sigma}=\left\{y_{\sigma} \in L^{2}\left(0, T ; Y_{h}\right): y_{\sigma \mid I_{k}} \in Y_{h} \forall k=1, \ldots, N_{\tau}\right\} .
\end{aligned}
$$

The elements of $\mathcal{Y}_{\sigma}$ can be written as

$$
y_{\sigma}=\sum_{k=1}^{N_{\tau}} y_{h, k} \chi_{k}=\sum_{k=1}^{N_{\tau}} \sum_{i=1}^{N_{I, h}} y_{i, k} e_{i} \chi_{k}
$$

where $y_{h, k} \in Y_{h}$ for $k=1, \ldots, N_{\tau}, y_{i, k} \in \mathbb{R}$ for $i=1, \ldots, N_{I, h}$, and $k=1, \ldots, N_{\tau}$, $\left\{e_{i}\right\}_{i=1}^{N_{I, h}}$ is the nodal basis associated to the interior nodes $\left\{x_{i}\right\}_{i=1}^{N_{I, h}}$ of the triangulation, and $\chi_{k}$ denotes the characteristic function of the interval $I_{k}=\left(t_{k-1}, t_{k}\right)$. For every $u \in L^{1}(0, T)^{m}$, we define its associated discrete state as the unique element 
$y_{\sigma}(u) \in \mathcal{Y}_{\sigma}$ such that

$$
\begin{gathered}
\int_{\Omega}\left(y_{h, k}-y_{h, k-1}\right) z_{h} d x+\tau_{k} a\left(y_{h, k}, z_{h}\right)+\int_{I_{k}} \int_{\Omega} f\left(x, t, y_{h, k}\right) z_{h} d x d t \\
=\sum_{j=1}^{m} \int_{I_{k}} \int_{\omega_{j}} u_{j}(t) g_{j}(x) z_{h} d x d t \quad \forall z_{h} \in Y_{h} \text { and all } k=1, \ldots, N_{\tau}, \\
\int_{\Omega} y_{h, 0} z_{h} d x=\int_{\Omega} y_{0} z_{h} d x \quad \forall z_{h} \in Y_{h},
\end{gathered}
$$

where

$$
a(y, z)=\sum_{i, j=1}^{n} \int_{\Omega} a_{i j} \partial_{x_{i}} y \partial_{x_{j}} z d x \quad \forall y, z \in H^{1}(\Omega) .
$$

From a computational point of view, this scheme can be interpreted as an implicit Euler discretization of the system of ordinary differential equations obtained after spatial finite element discretization. The proof of the existence and uniqueness of a solution for (4.3) is standard assuming that $\tau\left|C_{f}\right|<1$ with $C_{f}$ given by (1.2).

From [22, Corollaries 6.2 and 6.4], we have that for all $u \in U_{\mathrm{ad}}$

$$
\begin{aligned}
\left\|y_{\sigma}(u)-y_{u}\right\|_{L^{2}(Q)} & \leq C\left(h^{2}+\tau\right), \\
\left\|y_{\sigma}(u)-y_{u}\right\|_{L^{\infty}\left(0, T ; L^{2}(\Omega)\right)} & \leq C\left(h^{2}+\tau\right)|\log \tau|^{2} .
\end{aligned}
$$

The control is discretized using piecewise constant functions. Consider

$$
U_{\tau}=\left\{u_{\tau} \in L^{1}(0, T)^{m}: u_{\tau \mid I_{k}} \in \mathbb{R}^{m} \forall k=1, \ldots, N_{\tau}\right\} .
$$

We denote $\pi_{h}$ the Lagrange nodal interpolation operator in space, $\pi_{\tau}$ the $L^{2}(0, T)$ projection onto $U_{\tau}$, and $\pi_{\sigma}=\pi_{h} \circ \pi_{\tau}=\pi_{\tau} \circ \pi_{h}$. Notice that for all $u \in L^{1}(0, T)^{m}$ we have that

$$
y_{\sigma}(u)=y_{\sigma}\left(\pi_{\tau} u\right) \text { and } J_{\sigma}(u)=J_{\sigma}\left(\pi_{\tau} u\right) .
$$

Notice that if $u \in L^{1}(0, T)^{m} \backslash L^{2}(0, T)^{m}, \pi_{\tau} u$ is still well defined by the expression

$$
\left(\pi_{\tau} u\right)_{\left.\right|_{I_{k}}}=\frac{1}{\tau_{k}} \int_{I_{k}} u(t) d t, \quad k=1, \ldots, N_{\tau} .
$$

We formulate the discrete problem as

$$
\left(\mathrm{P}_{\sigma}\right) \min _{u_{\tau} \in U_{\tau, \mathrm{ad}}} J_{\sigma}\left(u_{\tau}\right),
$$

where

$$
J_{\sigma}(u)=\int_{Q} L\left(x, t, y_{\sigma}(u)(x, t)\right) d x d t,
$$

and $U_{\tau, \text { ad }}=U_{\tau} \cap U_{\mathrm{ad}}$. Since this set is compact and nonempty, the existence of a global solution of $\left(\mathrm{P}_{\sigma}\right)$ follows immediately from the continuity of $J_{\sigma}$ in $U_{\tau}$.

For every $u \in L^{1}(0, T)^{m}$, we define the related discrete adjoint state $\varphi_{\sigma}(u) \in \mathcal{Y}_{\sigma}$ as the unique solution of

$$
\begin{aligned}
\int_{\Omega}\left(\varphi_{h, k}-\varphi_{h, k+1}\right) z_{h} d x+\tau_{k} a\left(z_{h}, \varphi_{h, k}\right)+\int_{I_{k}} \int_{\Omega} \frac{\partial f}{\partial y}\left(x, t, y_{h, k}\right) \varphi_{h, k} z_{h} d x d t \\
=\int_{I_{k}} \int_{\Omega} \frac{\partial L}{\partial y}\left(x, t, y_{h, k}\right) z_{h} d x d t \quad \forall z_{h} \in Y_{h} \text { and all } k=N_{\tau}, \ldots, 1, \\
\varphi_{h, N_{\tau}+1}=0
\end{aligned}
$$

Copyright $@$ by SIAM. Unauthorized reproduction of this article is prohibited. 
where the functions $y_{h, k} \in \mathcal{Y}_{\sigma}$ are defined by $y_{\sigma}(u)=\sum_{k=1}^{N_{\tau}} y_{h, k} \chi_{k}$. We also define $\psi_{\sigma}(u) \in U_{\tau}$ as

$$
\psi_{\sigma, j}(u)(t)=\int_{\Omega} \varphi_{\sigma}(u)(x, t) g_{j}(x) d x \text { for } 1 \leq j \leq m
$$

With this notation, we have that for every $u, v \in L^{1}(0, T)^{m}$

$$
J_{\sigma}^{\prime}(u) v=\sum_{j=1}^{m} \int_{0}^{T} \psi_{\sigma, j}(u)(t) v_{j}(t) d t
$$

and first order conditions read as follows.

TheOrem 4.2. Suppose $\bar{u}_{\sigma} \in U_{\tau, \text { ad }}$ is a local solution of $\left(\mathrm{P}_{\sigma}\right)$ with associated discrete state $\bar{y}_{\sigma}=y_{\sigma}\left(\bar{u}_{\sigma}\right)$ and discrete adjoint state $\bar{\varphi}_{\sigma}=\varphi_{\sigma}\left(\bar{u}_{\sigma}\right)$. Then

$$
\int_{0}^{T} \bar{\psi}_{\sigma, j}(t)\left(u_{\tau, j}(t)-\bar{u}_{\sigma, j}(t)\right) d t \geq 0 \forall u_{\tau} \in U_{\tau, \text { ad }}, 1 \leq j \leq m,
$$

where $\bar{\psi}_{\sigma}=\psi_{\sigma}\left(\bar{u}_{\sigma}\right)$.

Analogously to (3.2), we can deduce that

$$
\bar{u}_{\sigma, j \mid I_{k}}=\left\{\begin{array} { l l } 
{ \alpha _ { j } } & { \text { if } \overline { \psi } _ { \sigma , j | I _ { k } } > 0 , } \\
{ \beta _ { j } } & { \text { if } \overline { \psi } _ { \sigma , j | I _ { k } } < 0 }
\end{array} \text { and } \overline { \psi } _ { \sigma , j | I _ { k } } \left\{\begin{array}{ll}
\geq 0 & \text { if } \bar{u}_{\sigma, j \mid I_{k}}=\alpha_{j}, \\
\leq 0 & \text { if } \bar{u}_{\sigma, j \mid I_{k}}=\beta_{j}, \\
=0 & \text { if } \alpha_{j}<\bar{u}_{\sigma, j \mid I_{k}}<\beta_{j}
\end{array}\right.\right.
$$

5. Error estimates for the optimal states. In this section, we first analyze the convergence of the approximations $\left(\mathrm{P}_{\sigma}\right)$ of $(\mathrm{P})$ in a sense to be made precise below. Then we prove error estimates for the difference between the discrete and the continuous optimal states.

THEOREM 5.1. Let $\bar{u}$ be a strict strong local minimizer for $(\mathrm{P})$, i.e.,

$$
\exists \rho>0: J(\bar{u})<J(u) \quad \forall u \in U_{\mathrm{ad}} \backslash\{\bar{u}\}:\left\|y_{u}-\bar{y}\right\|_{L^{\infty}(Q)} \leq \rho .
$$

Then, there exists a sequence $\left\{\bar{u}_{\sigma}\right\}_{\sigma}$ of local minimizers of $\left(\mathrm{P}_{\sigma}\right)$ such that $\bar{u}_{\sigma} \rightarrow \bar{u}$ weakly* in $L^{\infty}(0, T)^{m}$. Moreover,

$$
\exists \sigma_{0}: J_{\sigma}\left(\bar{u}_{\sigma}\right) \leq J_{\sigma}\left(u_{\tau}\right) \forall u_{\tau} \in U_{\tau, \text { ad }} \text { with }\left\|y_{\sigma}\left(u_{\tau}\right)-\bar{y}_{\sigma}\right\|_{L^{\infty}(Q)} \leq \frac{\rho}{2}, \forall|\sigma| \leq\left|\sigma_{0}\right|
$$

holds. Conversely, let $\left\{\bar{u}_{\sigma}\right\}_{\sigma}$ be a sequence of local minimizers of $\left(\mathrm{P}_{\sigma}\right)$ satisfying (5.2) for some given $\rho>0$ and such that $\bar{u}_{\sigma} \stackrel{*}{\rightarrow} \bar{u}$ in $L^{\infty}(0, T)^{m}$. Then $\bar{u}$ is a strong local solution of $(\mathrm{P})$ such that

$$
J(\bar{u}) \leq J(u) \quad \forall u \in U_{\mathrm{ad}}:\left\|y_{u}-\bar{y}\right\|_{L^{\infty}(Q)}<\frac{\rho}{2} .
$$

Before proving this theorem, we establish some auxiliary results.

Lemma 5.2. There exists $\mu \in(0,1)$ and $C_{\mu}$ such that

$$
\left\|y_{u}-\pi_{\sigma} y_{u}\right\|_{L^{\infty}(Q)} \leq C_{\mu}\left(h^{\mu}+\tau^{\mu / 2}\right) \quad \forall u \in U_{\text {ad }} .
$$

Copyright (c) by SIAM. Unauthorized reproduction of this article is prohibited. 
Proof. First, we observe that $H^{2}(\Omega) \subset C^{1 / 2}(\bar{\Omega})$ for $d \leq 3$. Then, according to [20, Theorem III-10.1], Theorem 2.1, and assumptions (A2), (H2), and (H3), we infer the existence of $\mu \in(0,1)$ and $C_{\mu}>0$ such that $y_{u} \in C^{\mu, \mu / 2}(\bar{Q})$ and

$$
\left\|y_{u}\right\|_{C^{\mu, \mu / 2}(\bar{Q})} \leq C_{\mu} \quad \forall u \in U_{\mathrm{ad}} .
$$

Now, given $\left(x_{0}, t_{0}\right)$ a point in a $K \times I_{k}$, we have with the Hölder continuity of $y_{u}$

$$
\begin{aligned}
& \left|y_{u}\left(x_{0}, t_{0}\right)-\pi_{\sigma} y_{u}\left(x_{0}, t_{0}\right)\right| \\
& \quad \leq\left|y_{u}\left(x_{0}, t_{0}\right)-\pi_{\tau} y_{u}\left(x_{0}, t_{0}\right)\right|+\left|\pi_{\tau}\left[y_{u}-\pi_{h} y_{u}\right]\left(x_{0}, t_{0}\right)\right| \\
& \quad \leq \frac{1}{\tau_{k}} \int_{I_{k}}\left|y_{u}\left(x_{0}, t_{0}\right)-y_{u}\left(x_{0}, t\right)\right| d t+\frac{1}{\tau_{k}} \int_{I_{k}}\left|y_{u}\left(x_{0}, t\right)-\pi_{h} y_{u}\left(x_{0}, t\right)\right| d t \\
& \quad \leq \frac{C_{\mu}}{\tau_{k}}\left(\int_{I_{k}}\left|t_{0}-t\right|^{\mu / 2} d t+\int_{I_{k}} h^{\mu} d t\right) \leq C_{\mu}\left(\tau^{\mu / 2}+h^{\mu}\right),
\end{aligned}
$$

which implies (5.4) due to the arbitrary selection of $\left(x_{0}, t_{0}\right)$.

COROllary 5.3. There exists a constant $M_{\mu}$ such that

$$
\left\|y_{\sigma}(u)-y_{u}\right\|_{L^{\infty}(Q)} \leq M_{\mu} \log \left(\frac{T}{\tau}\right)|\log h|\left(h^{\mu}+\tau^{\mu / 2}\right) \quad \forall u \in U_{\mathrm{ad}} .
$$

Proof. From [22, Theorem 6.5] we know that

$$
\left\|y_{\sigma}(u)-y_{u}\right\|_{L^{\infty}(Q)} \leq C \log \left(\frac{T}{\tau}\right)|\log h|\left\|y_{u}-y_{\sigma}\right\|_{L^{\infty}(Q)} \quad \forall y_{\sigma} \in \mathcal{Y}_{\sigma}, \quad \forall u \in U_{\mathrm{ad}} .
$$

Then it is enough to combine this inequality with (5.3) to deduce (5.6).

LEMma 5.4. Consider a sequence $\left\{u_{n}\right\}_{n} \subset U_{\text {ad }}$ such that $u_{n} \stackrel{*}{\rightarrow} u$ in $L^{\infty}(0, T)^{m}$ and a sequence $\left\{\sigma_{n}\right\}_{n}$ with $\left|\sigma_{n}\right|=h_{n}+\tau_{n} \rightarrow 0$. Then, $J_{\sigma_{n}}\left(u_{n}\right) \rightarrow J(u)$ holds.

Proof. We first write

$$
\left|J_{\sigma_{n}}\left(u_{n}\right)-J(u)\right| \leq\left|J_{\sigma_{n}}\left(u_{n}\right)-J\left(u_{n}\right)\right|+\left|J\left(u_{n}\right)-J(u)\right| .
$$

For the first term, by the mean value theorem, we know that there exists a measurable function $0<\theta_{n}(x, t)<1$ such that, if we name $\hat{y}_{n}=y_{\sigma_{n}}\left(u_{n}\right)+\theta_{n}\left(y_{u_{n}}-y_{\sigma_{n}}\left(u_{n}\right)\right)$, then using (4.4), (H3), and (A2) we obtain

$$
\begin{aligned}
& \left|J\left(u_{n}\right)-J_{\sigma_{n}}\left(u_{n}\right)\right| \\
& \quad=\left|\int_{0}^{T} \int_{\Omega}\left(L\left(x, t, y_{u_{n}}(x, t)\right)-L\left(x, t, y_{\sigma_{n}}\left(u_{n}\right)(x, t)\right)\right) d x d t\right| \\
& \quad \leq \int_{0}^{T} \int_{\Omega}\left|\frac{\partial L}{\partial y}\left(x, t, \hat{y}_{n}(x, t)\right)\left(y_{u_{n}}(x, t)-y_{\sigma_{n}}\left(u_{n}\right)(x, t)\right)\right| d x d t \\
& \quad \leq\left\|\frac{\partial L}{\partial y}\left(\cdot, \cdot, \hat{y}_{n}\right)\right\|_{L^{2}(Q)}\left\|y_{u_{n}}-y_{\sigma_{n}}\left(u_{n}\right)\right\|_{L^{2}(Q)} \leq C\left(h_{n}^{2}+\tau_{n}\right) .
\end{aligned}
$$

The convergence to zero of the second term follows from assumption (A2) and Lemma 2.2.

Proof of Theorem 5.1. Part I: Consider the set

$$
V_{a d, \sigma, \rho}=\left\{u_{\tau} \in U_{\tau, \text { ad }}:\left\|y_{\sigma}\left(u_{\tau}\right)-\bar{y}\right\|_{L^{\infty}(Q)} \leq \rho\right\} .
$$

Copyright (c) by SIAM. Unauthorized reproduction of this article is prohibited. 
From Corollary 5.3 we deduce the existence of $\sigma_{1}$ such that $\left\|y_{\sigma}(\bar{u})-\bar{y}\right\|_{L^{\infty}(Q)} \leq \rho$ for every $|\sigma| \leq\left|\sigma_{1}\right|$. Since $y_{\sigma}(\bar{u})=y_{\sigma}\left(\pi_{\tau} \bar{u}\right)$ and obviously $\pi_{\tau} \bar{u} \in U_{\tau, \text { ad }}$, we conclude that $\pi_{\tau} \bar{u} \in V_{a d, \sigma, \rho}$ for all $|\sigma| \leq\left|\sigma_{1}\right|$. Hence, $V_{a d, \sigma, \rho}$ is compact and nonempty. Therefore the problem

$$
\min _{u_{\tau} \in V_{a d, \sigma, \rho}} J_{\sigma}\left(u_{\tau}\right)
$$

has a solution $\bar{u}_{\sigma}$. We can extract a subsequence, denoted in the same way, such that $\bar{u}_{\sigma} \stackrel{*}{\rightarrow} \tilde{u}$ in $L^{\infty}(0, T)^{m}$. Since $U_{\tau, \text { ad }} \subset U_{\text {ad }}$ and $U_{\text {ad }}$ is weakly* closed in $L^{\infty}(0, T)^{m}$, $\tilde{u} \in U_{\text {ad }}$. We also have that $\bar{y}_{\sigma}=y_{\sigma}\left(\bar{u}_{\sigma}\right) \rightarrow y_{\tilde{u}}$ in $L^{\infty}(Q)$. To check this we write

$$
\left\|\bar{y}_{\sigma}-y_{\tilde{u}}\right\|_{L^{\infty}(Q)} \leq\left\|y_{\sigma}\left(\bar{u}_{\sigma}\right)-y_{\bar{u}_{\sigma}}\right\|_{L^{\infty}(Q)}+\left\|y_{\bar{u}_{\sigma}}-y_{\tilde{u}}\right\|_{L^{\infty}(Q)} .
$$

From Corollary 5.3 and Lemma 2.2 we infer that both terms converge to 0 . Since $\bar{u}_{\sigma} \in V_{a d, \sigma, \rho}$, we have that

$$
\begin{aligned}
\left\|y_{\tilde{u}}-\bar{y}\right\|_{L^{\infty}(Q)} & \leq\left\|y_{\tilde{u}}-y_{\sigma}\left(\bar{u}_{\sigma}\right)\right\|_{L^{\infty}(Q)}+\left\|y_{\sigma}\left(\bar{u}_{\sigma}\right)-\bar{y}\right\|_{L^{\infty}(Q)} \\
& \leq\left\|y_{\tilde{u}}-y_{\sigma}\left(\bar{u}_{\sigma}\right)\right\|_{L^{\infty}(Q)}+\rho \rightarrow \rho \quad \text { as }|\sigma| \rightarrow 0 .
\end{aligned}
$$

Passing to the limit in $J_{\sigma}\left(\bar{u}_{\sigma}\right) \leq J_{\sigma}\left(\pi_{\tau} \bar{u}\right)$ with Lemma 2.2 , we infer that $J(\tilde{u}) \leq J(\bar{u})$. Due to (5.1), this is possible only if $\tilde{u}=\bar{u}$, and so (5.7) implies that $\left\|\bar{y}_{\sigma}-\bar{y}\right\|_{L^{\infty}(Q)} \rightarrow 0$ as $|\sigma| \rightarrow 0$. Let us take $\sigma_{0}$ such that $\left\|\bar{y}_{\sigma}-\bar{y}\right\|_{L^{\infty}(Q)}<\rho / 2$. Then for any $u_{\tau} \in U_{\tau \text {,ad }}$ such that $\left\|y_{\sigma}\left(u_{\tau}\right)-\bar{y}_{\sigma}\right\|_{L^{\infty}(Q)} \leq \rho / 2$, we get

$$
\left\|y_{\sigma}\left(u_{\tau}\right)-\bar{y}\right\|_{L^{\infty}(Q)} \leq\left\|y_{\sigma}\left(u_{\tau}\right)-\bar{y}_{\sigma}\right\|_{L^{\infty}(Q)}+\left\|\bar{y}_{\sigma}-\bar{y}\right\|_{L^{\infty}(Q)}<\rho \quad \forall|\sigma| \leq\left|\sigma_{0}\right| .
$$

Then $u_{\tau} \in V_{a d, \sigma, \rho}$ and, hence, $J\left(\bar{u}_{\sigma}\right) \leq J\left(u_{\tau}\right)$ for every $|\sigma| \leq\left|\sigma_{0}\right|$, which proves the first part of the theorem.

Part II: We denote, as above, $\bar{y}_{\sigma}$ and $\bar{y}$ the discrete and continuous states associated with $\bar{u}_{\sigma}$ and $\bar{u}$, respectively. We observe that, proceeding as in (5.7), $\| \bar{y}_{\sigma}-$ $\bar{y} \|_{L^{\infty}(Q)} \rightarrow 0$ as $|\sigma| \rightarrow 0$. Let us take an arbitrary element $u \in U_{\text {ad }}$ such that $\left\|y_{u}-\bar{y}\right\|_{L^{\infty}(Q)}<\rho / 2$. We have to prove that $J(\bar{u}) \leq J(u)$. To this end, we consider the discrete controls $\pi_{\tau} u$. It is obvious that $\pi_{\tau} u \in U_{\tau, \text { ad }}$ and, from Corollary 5.3 and the fact that $y_{\sigma}\left(\pi_{\tau} u\right)=y_{\sigma}(u)$ (see (4.6)), we have that $\left\|y_{\sigma}\left(\pi_{\tau} u\right)-y_{u}\right\|_{L^{\infty}(Q)} \rightarrow 0$ as $|\sigma| \rightarrow 0$. Therefore, we get

$$
\left\|y_{\sigma}\left(\pi_{\tau} u\right)-\bar{y}_{\sigma}\right\|_{L^{\infty}(Q)} \rightarrow\left\|y_{u}-\bar{y}\right\|_{L^{\infty}(Q)}<\frac{\rho}{2} \text { as } \sigma \rightarrow 0 .
$$

Hence, there exists $\sigma_{1}$ with $\left|\sigma_{1}\right| \leq\left|\sigma_{0}\right|$ such that

$$
\left\|y_{\sigma}\left(\pi_{\tau} u\right)-\bar{y}_{\sigma}\right\|_{L^{\infty}(Q)}<\frac{\rho}{2} \quad \forall|\sigma| \leq\left|\sigma_{1}\right| .
$$

Thus, from (5.2) and Lemma 5.4 we infer

$$
J(\bar{u})=\lim _{\sigma \rightarrow 0} J_{\sigma}\left(\bar{u}_{\sigma}\right) \leq \lim _{\sigma \rightarrow 0} J_{\sigma}\left(\pi_{\tau} u\right)=J(u),
$$

which concludes the proof.

Remark 5.5. Let us observe that if $\left\{\bar{u}_{\sigma}\right\}_{\sigma}$ is a sequence of global minimizers of $\left(\mathrm{P}_{\sigma}\right)$, then there exist subsequences converging to elements $\bar{u}$. Any of these controls $\bar{u}$ is a global minimizer of $(\mathrm{P})$. This is an immediate consequence of the second part of Theorem 5.1. Indeed, it is enough to take $\rho$ sufficiently large. 
Assuming the second order optimality conditions we can prove some error estimates for the difference between the continuous and discrete optimal states.

THEOREM 5.6. Let $\bar{u}$ be a local solution of $(\mathrm{P})$ satisfying the first order conditions (3.1a)-(3.1c) and the second order sufficient conditions (3.3). Let $\left\{\bar{u}_{\sigma}\right\}_{\tau}$ be a sequence of local minima of $\left(\mathrm{P}_{\sigma}\right)$ such that (5.2) holds and $\bar{u}_{\sigma} \stackrel{*}{\rightarrow} \bar{u}$ in $L^{\infty}(0, T)^{m}$. Then, there exists a constant $C$ such that

$$
\left\|\bar{y}_{\sigma}-\bar{y}\right\|_{L^{2}(Q)} \leq C(h+\sqrt{\tau}) .
$$

Proof. By the triangle inequality we have

$$
\left\|\bar{y}_{\sigma}-\bar{y}\right\|_{L^{2}(Q)} \leq\left\|\bar{y}_{\sigma}-y_{\bar{u}_{\sigma}}\right\|_{L^{2}(Q)}+\left\|y_{\bar{u}_{\sigma}}-\bar{y}\right\|_{L^{2}(Q)} .
$$

The first term in the right-hand side is of order $O\left(h^{2}+\tau\right)$; see (4.4). We just need to study the second term. From Lemma 2.2 we know that $y_{\bar{u}_{\sigma}} \rightarrow \bar{y}$ strongly in $L^{\infty}(Q)$. From Theorem 3.4, we deduce the existence of $\varepsilon>0$ and $\kappa>0$ such that (3.6) holds. Then, there exists $\sigma_{0}>0$ such that for all $0<|\sigma|<\left|\sigma_{0}\right|,\left\|y_{\bar{u}_{\sigma}}-\bar{y}\right\|_{L^{\infty}(Q)}<\varepsilon$. Thus, using (3.6) we have

$$
\begin{aligned}
\frac{\kappa}{2}\left\|y_{\bar{u}_{\sigma}}-\bar{y}\right\|_{L^{2}(Q)}^{2} \leq & J\left(\bar{u}_{\sigma}\right)-J(\bar{u}) \\
\leq & {\left[J\left(\bar{u}_{\sigma}\right)-J_{\sigma}\left(\bar{u}_{\sigma}\right)\right]+\left[J_{\sigma}\left(\bar{u}_{\sigma}\right)-J_{\sigma}\left(\pi_{\tau} \bar{u}\right)\right] } \\
& +\left[J_{\sigma}\left(\pi_{\tau} \bar{u}\right)-J_{\sigma}(\bar{u})\right]+\left[J_{\sigma}(\bar{u})-J(\bar{u})\right] \\
= & \mathrm{I}+\mathrm{II}+\mathrm{III}+\mathrm{IV} .
\end{aligned}
$$

Let us estimate the first term. By the mean value theorem, there exists a measurable function $\hat{y}_{\sigma}=\bar{y}_{\sigma}+\theta\left(y_{\bar{u}_{\sigma}}-\bar{y}_{\sigma}\right)$ with $0<\theta(x, t)<1$ such that

$$
\begin{aligned}
J\left(\bar{u}_{\sigma}\right)-J_{\sigma}\left(\bar{u}_{\sigma}\right) & =\int_{Q}\left(L\left(x, t, y_{\bar{u}_{\sigma}}(x, t)\right)-L\left(x, t, \bar{y}_{\sigma}(x, t)\right)\right) d x d t \\
& =\int_{Q} \frac{\partial L}{\partial y}\left(x, t, \hat{y}_{\sigma}(x, t)\right)\left(y_{\bar{u}_{\sigma}}(x, t)-\bar{y}_{\sigma}(x, t)\right) d x d t \\
& \leq\left\|\frac{\partial L}{\partial y}\left(\cdot, \cdot, \hat{y}_{\sigma}\right)\right\|_{L^{2}(Q)}\left\|y_{\bar{u}_{\sigma}}-\bar{y}_{\sigma}\right\|_{L^{2}(Q)} \leq C\left(h^{2}+\tau\right),
\end{aligned}
$$

where we have used (A2), Theorem 2.1, and (4.4) for the last two inequalities. The fourth term can be estimated exactly in the same way.

Since $\bar{u}_{\sigma}$ satisfies (5.2), we have that II $\leq 0$ for $|\sigma|$ small enough. Indeed, we can argue as in the second part of the proof of Theorem 5.1 to deduce the existence of $\sigma_{1}$ such that $\pi_{\tau} \bar{u} \in U_{\tau, \text { ad }}$ and $\left\|y_{\sigma}\left(\pi_{\tau} \bar{u}\right)-\bar{y}_{\sigma}\right\|_{L^{\infty}(Q)}<\rho / 2$ for $|\sigma|<\left|\sigma_{1}\right|$. Finally, the term III is zero because of (4.6).

Collecting all the estimates, we achieve the desired result.

6. Bang-bang control and control error estimates. In the last section we have used the quadratic growth property of the states (3.6) to prove error estimates between discrete and continuous optimal states. The reader could be wondering if it is possible to get an analogous condition involving a quadratic term for the controls. The answer is negative in general. In [16], the authors prove that if $\bar{u}$ is a local minimizer of $(\mathrm{P})$, which is not bang-bang, then there do not exist $\varepsilon>0, \kappa>0$, $\gamma>0$, and $r \geq 1$ such that the inequality

$$
J(\bar{u})+\frac{\kappa}{2}\|u-\bar{u}\|_{L^{r}(0, T)^{m}}^{\gamma} \leq J(u) \quad \forall u \in U_{\mathrm{ad}}:\|u-\bar{u}\|_{L^{1}(0, T)^{m}} \leq \varepsilon
$$

Copyright $@$ by SIAM. Unauthorized reproduction of this article is prohibited. 
holds. However, if we make a certain structural assumption on the associated adjoint state with $\bar{u}$, which implies the bang-bang property of $\bar{u}$, then we can get the desired inequality. Following [16], the next hypothesis will be assumed in the rest of the paper.

$$
\exists K>0, \exists \gamma \in(0,1]: \text { meas }\left\{t \in[0, T]:\left|\bar{\psi}_{j}(t)\right| \leq \varepsilon\right\} \leq K \varepsilon^{\gamma} \forall \varepsilon>0,1 \leq j \leq m .
$$

Remark 6.1. Notice that under this assumption $\bar{u}$ is a bang-bang control. Let us comment on this. Assumption (6.1) rules out the possibility of having meas $\{t \in$ $\left.[0, T]: \bar{\psi}_{j}(t)=0\right\}>0$, so we will not have singular arcs.

If $\bar{\psi}_{j}$ is regular enough and there is a finite number of points $\left\{t_{k}\right\}_{k=1}^{N}$ such that $\bar{\psi}_{j}^{(n)}\left(t_{k}\right)=0$ for $0 \leq n<m_{k}$ and $\bar{\psi}_{j}^{\left(m_{k}\right)}\left(t_{k}\right) \neq 0$, then (6.1) is fulfilled by $\bar{\psi}_{j}$ with $\gamma=\min \left\{1 / m_{k}: k=1, \ldots, N\right\}$.

Suppose now that $\bar{\psi}_{j} \in C^{1}[0, T]$. Then, (6.1) holds with $\gamma=1$ if and only if the number of points where $\bar{\psi}_{j}$ vanishes is finite and the derivatives at these points are not zero. To prove this, first we suppose that there is an infinite number of points in $[0, T]$ where $\psi_{j}$ vanishes. Then, we can extract a sequence $\left\{t_{n}\right\}_{n=1}^{\infty} \subset[0, T]$ converging to some point $t_{0} \in[0, T]$. By continuity of $\psi_{j}, \bar{\psi}_{j}\left(t_{0}\right)=0$. By the mean value theorem, between every two consecutive terms of this sequence there is a point $t_{n}^{*}$ such that $\bar{\psi}_{j}^{\prime}\left(t_{n}^{*}\right)=0$, and hence $\bar{\psi}_{j}^{\prime}\left(t_{0}\right)=0$. In a neighborhood of $t_{0}$, we have that $\left|\psi_{j}(t)\right|=o\left(t-t_{0}\right)$. This contradicts $(6.1)$.

The converse implication follows from the first part of this remark.

Next we prove that, under the assumption (6.1), the sufficient second order condition (3.3) leads to a better growth condition than the one given in (3.6). Actually, this new growth condition involves also the controls. Before stating this result, we establish a lemma whose proof is the same as the one of [24, Proposition 3.2] with the obvious changes; see also [16, Proposition 2.7] for the case $\gamma=1$.

Lemma 6.2. Let $\bar{u} \in U_{\mathrm{ad}}$ satisfy (3.1a)-(3.1c) and (6.1), and then

$$
J^{\prime}(\bar{u})(u-\bar{u}) \geq \nu\|u-\bar{u}\|_{L^{1}(0, T)^{m}}^{1+\frac{1}{\gamma}} \quad \forall u \in U_{\mathrm{ad}},
$$

where $\nu=\frac{1}{2}\left(2 K \sum_{j=1}^{m}\left(\beta_{j}-\alpha_{j}\right)\right)^{-1 / \gamma}$.

THEOREM 6.3. Under the assumptions and notation of Lemma 6.2 and supposing that $\bar{u}$ satisfies the sufficient second order condition (3.3), then there exist $\varepsilon>0$ and $\kappa>0$ such that

$$
J(\bar{u})+\frac{\nu}{2}\|u-\bar{u}\|_{L^{1}(0, T)^{m}}^{1+\frac{1}{\gamma}}+\frac{\kappa}{2}\left\|y_{u}-\bar{y}\right\|_{L^{2}(Q)}^{2} \leq J(u) \forall u \in U_{\mathrm{ad}}:\left\|y_{u}-\bar{y}\right\|_{L^{\infty}(Q)}<\varepsilon
$$

where $\bar{y}$ is the state associates with $\bar{u}$. follows:

Proof. To prove (6.3) it is enough to make a Taylor expansion and to use (6.2) as

$$
\begin{aligned}
J(u) & =J(\bar{u})+\frac{1}{2} J^{\prime}(\bar{u})(u-\bar{u})+\frac{1}{2}\left[J^{\prime}(\bar{u})(u-\bar{u})+J^{\prime \prime}(\bar{u}+\theta(u-\bar{u}))(u-\bar{u})^{2}\right] \\
& \geq J(\bar{u})+\frac{\nu}{2}\|u-\bar{u}\|_{L^{1}(0, T)^{m}}^{1+\frac{1}{\gamma}}+\frac{1}{2}\left[J^{\prime}(\bar{u})(u-\bar{u})+J^{\prime \prime}(\bar{u}+\theta(u-\bar{u}))(u-\bar{u})^{2}\right] .
\end{aligned}
$$

Now we can estimate the last term with (3.23) taking $\rho=1$.

Copyright (c) by SIAM. Unauthorized reproduction of this article is prohibited. 
Remark 6.4. In the case $\gamma=1$, it was proved in [15] (see also [16]) that the condition

$$
J^{\prime \prime}(\bar{u}) v^{2} \geq-\nu^{\prime}\|v\|_{L^{2}(0, T)^{m}}^{2} \quad \forall v \in C_{\bar{u}}^{\tau}
$$

with $0 \leq \nu^{\prime}<2 \nu$ implies the existence of $\varepsilon>0$ and $\kappa>0$ such that

$$
J(\bar{u})+\frac{\kappa}{2}\|u-\bar{u}\|_{L^{1}(0, T)^{m}}^{2} \leq J(u) \quad \forall u \in U_{\mathrm{ad}}:\|u-\bar{u}\|_{L^{1}(0, T)^{m}} \leq \varepsilon .
$$

Next, we consider the discrete control problems $\left(\mathrm{P}_{\sigma}\right)$ defined in section 4 . Let $\bar{u}$ be a local minimizer of $(\mathrm{P})$ satisfying the second order condition (3.3) and the assumption (6.1). Then, from Theorem 5.1 we get the existence of a sequence $\left\{\bar{u}_{\sigma}\right\}_{\sigma}$ of local minimizers of problems $\left(\mathrm{P}_{\sigma}\right)$ such that $\bar{u}_{\sigma} \stackrel{*}{\rightarrow} \bar{u}$ in $L^{\infty}(0, T)^{m},\left\|\bar{y}_{\sigma}-\bar{y}\right\|_{L^{\infty}(Q)} \rightarrow 0$ as $|\sigma| \rightarrow 0$, and (5.2) is fulfilled. In addition, since $\bar{u}$ is a bang-bang control, we have that $\bar{u}_{\sigma} \rightarrow \bar{u}$ strongly in $L^{p}(0, T)^{m}$ for every $p \in[1, \infty)$. Indeed, it is enough to observe that

$$
\int_{0}^{T}\left|\bar{u}_{\sigma, j}-\bar{u}_{j}\right| d t=\int_{I_{\alpha_{j}}}\left(\bar{u}_{\sigma, j}-\bar{u}_{j}\right) d t+\int_{I_{\beta_{j}}}\left(\bar{u}_{j}-\bar{u}_{\sigma, j}\right) d t \longrightarrow 0 \text { as } \tau \rightarrow 0,
$$

where

$$
I_{\alpha_{j}}=\left\{t \in(0, T): \bar{u}_{j}(t)=\alpha_{j}\right\} \text { and } I_{\beta_{j}}=\left\{t \in(0, T): \bar{u}_{j}(t)=\beta_{j}\right\} .
$$

This proves the strong convergence in $L^{1}(0, T)^{m}$. Moreover, due to the boundedness in $L^{\infty}(0, T)^{m}$ of the sequence $\left\{\bar{u}_{\sigma}\right\}_{\sigma}$, we conclude the strong convergence in $L^{p}(0, T)^{m}$ for every $p<\infty$.

The next theorem provides an estimate for the difference $\bar{u}_{\sigma}-\bar{u}$.

THEOREM 6.5. Under the previous notation and assumptions, there exists a constant independent of $\sigma$ such that

$$
\begin{aligned}
\left\|\bar{u}_{\sigma}-\bar{u}\right\|_{L^{1}(0, T)^{m}} & \leq C\left[\left(h^{2}+\tau\right)|\log \tau|^{2}\right]^{\gamma}, \\
\left\|\bar{y}_{\sigma}-\bar{y}\right\|_{L^{2}(Q)} & \leq C\left[\left(h^{2}+\tau\right)|\log \tau|^{2}\right]^{\frac{\gamma+1}{2}} .
\end{aligned}
$$

Proof. Since $\bar{u}_{\sigma} \rightarrow \bar{u}$, for any $\varepsilon>0$ there exists $\sigma_{0}>0$ such that $\left\|\bar{y}_{\sigma}-\bar{y}\right\|_{L^{\infty}(Q)}<\varepsilon$ for every $|\sigma|<\left|\sigma_{0}\right|$. Now, using (6.2) and the fact

$$
\begin{aligned}
J_{\sigma}^{\prime}\left(\bar{u}_{\sigma}\right)\left(\bar{u}-\bar{u}_{\sigma}\right) & =\sum_{j=1}^{m} \int_{0}^{T} \bar{\psi}_{\sigma, j}\left(\bar{u}_{j}-\bar{u}_{\sigma, j}\right) d t \\
& =\sum_{j=1}^{m} \int_{0}^{T} \bar{\psi}_{\sigma, j}\left(\pi_{\tau} \bar{u}_{j}-\bar{u}_{\sigma, j}\right) d t=J_{\sigma}^{\prime}\left(\bar{u}_{\sigma}\right)\left(\pi_{\tau} \bar{u}-\bar{u}_{\sigma}\right) \geq 0,
\end{aligned}
$$

we get with the mean value theorem the existence of $\theta \in(0,1)$ such that we have for $u_{\theta}=\bar{u}+\theta\left(\bar{u}-\bar{u}_{\sigma}\right)$

$$
\begin{aligned}
& \frac{\nu}{2}\left\|\bar{u}_{\sigma}-\bar{u}\right\|_{L^{1}(0, T)^{m}}^{1+\frac{1}{\gamma}}+\frac{1}{2} J^{\prime}(\bar{u})\left(\bar{u}_{\sigma}-\bar{u}\right) \\
& \quad \leq J^{\prime}(\bar{u})\left(\bar{u}_{\sigma}-\bar{u}\right) \leq\left[J^{\prime}(\bar{u})-J_{\sigma}^{\prime}\left(\bar{u}_{\sigma}\right)\right]\left(\bar{u}_{\sigma}-\bar{u}\right) \\
& \quad=\left[J^{\prime}(\bar{u})-J^{\prime}\left(\bar{u}_{\sigma}\right)\right]\left(\bar{u}_{\sigma}-\bar{u}\right)+\left[J^{\prime}\left(\bar{u}_{\sigma}\right)-J_{\sigma}^{\prime}\left(\bar{u}_{\sigma}\right)\right]\left(\bar{u}_{\sigma}-\bar{u}\right) \\
& \quad=-J^{\prime \prime}\left(u_{\theta}\right)\left(\bar{u}_{\sigma}-\bar{u}\right)^{2}+\sum_{j=1}^{m} \int_{0}^{T}\left(\psi_{\bar{u}_{\sigma}, j}-\bar{\psi}_{\sigma, j}\right)\left(\bar{u}_{j}-\bar{u}_{\sigma, j}\right) d t
\end{aligned}
$$

Copyright $\odot$ by SIAM. Unauthorized reproduction of this article is prohibited. 
Equivalently, we have

$$
\begin{gathered}
\frac{\nu}{2}\left\|\bar{u}_{\sigma}-\bar{u}\right\|_{L^{1}(0, T)^{m}}^{1+\frac{1}{\gamma}}+\left[\frac{1}{2} J^{\prime}(\bar{u})\left(\bar{u}_{\sigma}-\bar{u}\right)+J^{\prime \prime}\left(u_{\theta}\right)\left(\bar{u}_{\sigma}-\bar{u}\right)^{2}\right] \\
\left.\leq \sum_{j=1}^{m} \int_{0}^{T} \int_{\Omega}\left(\varphi_{\bar{u}_{\sigma}}-\bar{\varphi}_{\sigma}\right)\right) g_{j}\left(\bar{u}_{j}-\bar{u}_{\sigma, j}\right) d x d t .
\end{gathered}
$$

From Remark 3.6 we deduce the existence of $\lambda>0$ such that for $\varepsilon$ as above sufficiently small

$$
\frac{\lambda}{2}\left\|y_{\bar{u}_{\sigma}}-\bar{y}\right\|_{L^{2}(Q)}^{2} \leq \frac{1}{2} J^{\prime}(\bar{u})\left(\bar{u}_{\sigma}-\bar{u}\right)+J^{\prime \prime}\left(u_{\theta}\right)\left(\bar{u}_{\sigma}-\bar{u}\right)^{2} \quad \forall|\sigma| \leq\left|\sigma_{0}\right| .
$$

Let us estimate the right-hand side of (6.6). To this end, we introduce the function $\varphi^{\sigma} \in W^{1, p}\left(0, T ; L^{2}(\Omega)\right) \cap L^{p}\left(0, T ; H^{2}(\Omega)\right)$ for all $p<\infty$ as the solution of

$$
\left\{\begin{aligned}
-\frac{\partial \varphi}{\partial t}+A^{*} \varphi+\frac{\partial f}{\partial y}\left(x, t, \bar{y}_{\sigma}\right) \varphi & =\frac{\partial L}{\partial y}\left(x, t, \bar{y}_{\sigma}\right) \text { in } Q, \\
\varphi & =0 \text { on } \Sigma, \\
\varphi(T) & =0 \text { in } \Omega .
\end{aligned}\right.
$$

Obviously, estimate (4.5) can be applied to estimate $\varphi^{\sigma}-\bar{\varphi}_{\sigma}$, and hence we have

$$
\left\|\varphi^{\sigma}-\bar{\varphi}_{\sigma}\right\|_{L^{\infty}\left(0, T ; L^{2}(\Omega)\right.} \leq C_{1}\left(h^{2}+\tau\right)|\log \tau|^{2} .
$$

Now, we estimate the difference $z^{\sigma}=\varphi_{\bar{u}_{\sigma}}-\varphi^{\sigma}$. Subtracting the equations satisfied by $\varphi_{\bar{u}_{\sigma}}$ and $\varphi^{\sigma}$ we obtain

$$
\left\{\begin{array}{l}
-\frac{\partial z^{\sigma}}{\partial t}+A^{*} z^{\sigma}+\frac{\partial f}{\partial y}\left(x, t, y_{\bar{u}_{\sigma}}\right) z^{\sigma} \\
=\left[\frac{\partial f}{\partial y}\left(x, t, \bar{y}_{\sigma}\right)-\frac{\partial f}{\partial y}\left(x, t, y_{\bar{u}_{\sigma}}\right)\right] \varphi^{\sigma}+\left[\frac{\partial L}{\partial y}\left(x, t, y_{\bar{u}_{\sigma}}\right)-\frac{\partial L}{\partial y}\left(x, t, \bar{y}_{\sigma}\right)\right] \text { in } Q \\
z^{\sigma}=0 \text { on } \Sigma, \quad z^{\sigma}(T)=0 \text { in } \Omega .
\end{array}\right.
$$

From assumptions (A2) and (A5) and Theorem 2.1 and using the mean value theorem we infer

$$
\left\|z^{\sigma}\right\|_{L^{\infty}\left(0, T ; L^{2}(\Omega)\right)} \leq C_{2}\left\|y_{\bar{u}_{\sigma}}-\bar{y}_{\sigma}\right\|_{L^{2}(Q)} .
$$

Now, (4.4) and the definition of $z^{\sigma}$ imply

$$
\left\|\varphi_{\bar{u}_{\sigma}}-\varphi^{\sigma}\right\|_{L^{\infty}\left(0, T ; L^{2}(\Omega)\right)} \leq C_{3}\left(h^{2}+\tau\right) .
$$

Finally, applying (6.8) and (6.9) we obtain with Young's inequality for $p=\gamma+1$ and $q=(\gamma+1) / \gamma$

$$
\begin{aligned}
& \left.\sum_{j=1}^{m} \int_{0}^{T} \int_{\Omega}\left(\varphi_{\bar{u}_{\sigma}}-\bar{\varphi}_{\sigma}\right)\right) g_{j}\left(\bar{u}_{j}-\bar{u}_{\sigma, j}\right) d x d t \\
& \quad \leq \max _{1 \leq j \leq m}\left\|g_{j}\right\|_{L^{2}(\Omega)}\left\|\varphi_{\bar{u}_{\sigma}}-\bar{\varphi}_{\sigma}\right\|_{L^{\infty}\left(0, T ; L^{2}(\Omega)\right)}\left\|\bar{u}-\bar{u}_{\sigma}\right\|_{L^{1}(0, T)^{m}} \\
& \quad \leq C_{4}\left(h^{2}+\tau\right)|\log \tau|^{2}\left\|\bar{u}-\bar{u}_{\sigma}\right\|_{L^{1}(0, T)^{m}} \\
& \quad \leq C_{5}\left[\left(h^{2}+\tau\right)|\log \tau|^{2}\right]^{\gamma+1}+\frac{\nu}{4}\left\|\bar{u}-\bar{u}_{\sigma}\right\|_{L^{1}(0, T)^{m}}^{1+\frac{1}{\gamma}} .
\end{aligned}
$$

Copyright (c) by SIAM. Unauthorized reproduction of this article is prohibited. 
Finally, combining (6.6), (6.7), and (6.10) we get

$$
\frac{\nu}{4}\left\|\bar{u}_{\sigma}-\bar{u}\right\|_{L^{1}(0, T)^{m}}^{1+\frac{1}{\gamma}}+\frac{\lambda}{2}\left\|\bar{y}_{\sigma}-\bar{y}\right\|_{L^{2}(Q)}^{2} \leq C_{5}\left[\left(h^{2}+\tau\right)|\log \tau|^{2}\right]^{\gamma+1},
$$

which leads straightforwardly to (6.4) and (6.5).

7. Numerical experiment. Consider $\Omega=(0,1), T=1, A=-\partial_{x x}^{2}, y_{0}(x)=$ $x(1-x)$, and $m=1$. Define $\omega=[0.25,0.75]$ and $g(x)=\chi_{\omega}(x)$. We will take $f(x, t, y)=y^{3}+e(x, t)$ and $L(x, t, y)=y^{4}+b(x, t) y$, where $e(x, t)$ and $b(x, t)$ are defined later. Finally, we take the control constraints $\alpha=0$ and $\beta=1$.

The state equation is given by

$$
\left\{\begin{aligned}
\frac{\partial y}{\partial t}-\frac{\partial^{2} y}{\partial x^{2}}+y^{3}+e(x, t) & =u(t) g(x) \text { in } \Omega \times(0, T), \\
y(0, t)=y(1, t) & =0 \text { for } t \in(0, T) \\
y(x, 0) & =x(1-x) \text { for } x \in(0,1)
\end{aligned}\right.
$$

and the adjoint state equation is given by

$$
\left\{\begin{aligned}
-\frac{\partial \varphi}{\partial t}-\frac{\partial^{2} \varphi}{\partial x^{2}}+3 y^{2} \varphi & =4 y^{3}+b(x, t) \text { in } \Omega \times(0, T), \\
\varphi(0, t)=\varphi(1, t) & =0 \text { for } t \in(0, T), \\
\varphi(x, T) & =0 \text { for } x \in(0,1) .
\end{aligned}\right.
$$

We fix $\bar{y}(x, t)=x(1-x) \cos (2 \pi t)$, which satisfies the boundary and initial conditions of the state equation. For specific examples, we just need to define $\bar{\varphi}$ and $\bar{u}$. With these choices, we define

$$
e(x, t)=-\frac{\partial \bar{y}}{\partial t}+\frac{\partial^{2} \bar{y}}{\partial x^{2}}-\bar{y}^{3}+\bar{u}(t) g(x)
$$

and

$$
b(x, t)=-\frac{\partial \bar{\varphi}}{\partial t}-\frac{\partial^{2} \bar{\varphi}}{\partial x^{2}}+3 \bar{y}^{2} \varphi-4 \bar{y}^{3} .
$$

We have that $(\bar{u}, \bar{y}, \bar{\varphi})$ satisfies the first order optimality conditions (3.1a)-(3.1c).

To solve the problem we do a Tikhonov regularization (cf. [23]), i.e., we solve

$$
\min _{u \in U_{\mathrm{ad}}} J_{\nu_{j}}(u)=J(u)+\frac{\nu_{j}}{2}\|u\|_{L^{2}(0, T)^{m}}^{2}
$$

for a sequence $\nu_{j} \searrow 0$. This problem is solved using a semismooth Newton method as described in [10, section 14]. We use the following algorithm.

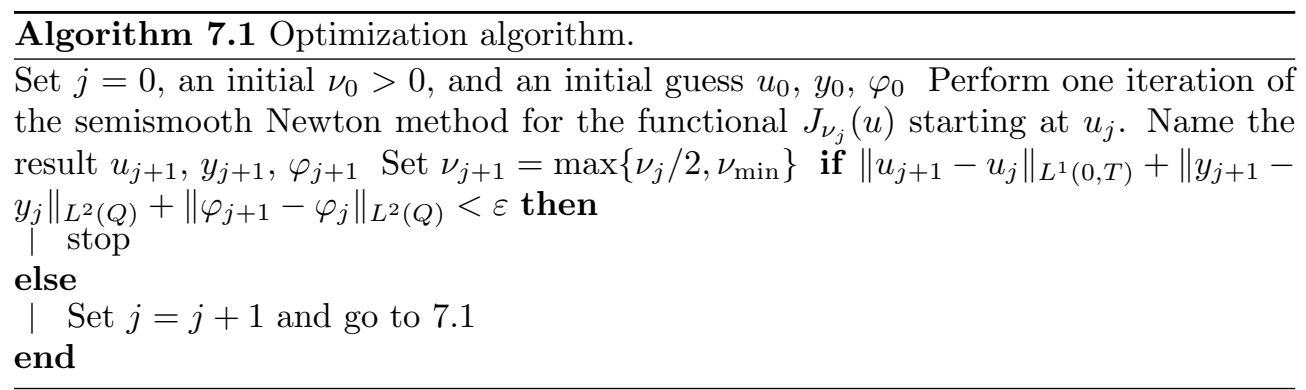

Copyright (C) by SIAM. Unauthorized reproduction of this article is prohibited. 
To discretize the problem, we use two families of uniform partitions in time and space. In time we take $\tau_{k}=3^{-k} T, k=k_{0}, \ldots, K$, so that $\bar{u} \notin U_{\tau}$. In space, we take $h_{i}=2^{-i}, i=i_{0}, \ldots, I$.

We measure the error in the state variable with respect to $\tilde{\pi}_{\tau_{k}} \Pi_{h_{i} / 2} \bar{y}$, where $\tilde{\pi}_{\tau}$ is the numerical approximation of $\pi_{\tau}$ given by

$$
\pi_{\tau} v=\sum_{j=1}^{N_{\tau}} \frac{1}{\tau} \int_{I_{j}} v(t) d t \chi_{j} \approx \tilde{\pi}_{\tau} v=\sum_{j=1}^{N_{\tau}} v\left(\frac{t_{j}+t_{j-1}}{2}\right) \chi_{j} \quad \forall v \in C[0, T],
$$

i.e., the midpoint quadrature formula is replacing the integral.

7.1. Bang-bang control. We define $\bar{\varphi}(x, t)=x(1-x) \sin (2 \pi t / T)$. This function clearly satisfies the boundary and end conditions of the adjoint state equation. For $T>0$ small enough, $L^{\prime \prime}(\bar{y})-\bar{\varphi} f^{\prime \prime}(y)>0$ in $Q$ and second order sufficient conditions hold.

We have that the switching function is

$$
\bar{\psi}(t)=\int_{0.25}^{0.75} \bar{\varphi}(x, t) d x=\frac{11}{96} \sin (2 \pi t / T) .
$$

So we define

$$
\bar{u}(t)=\left\{\begin{array}{lll}
0 & \text { if } & 0<t<0.5 T \\
1 & \text { if } \quad 0.5 T<t<T .
\end{array}\right.
$$

Assumption (6.1) is satisfied for $\gamma=1$.

We perform two experiments: In the first one, we fix a small time step $\tau=3^{-8} \mathrm{~T}$ and we measure the error $e_{i}^{h, y}$ in the state variable as the space mesh size varies. We have not been able to measure the dependence of the error in the control with respect to the spatial discretization parameter.

In the second one, we fix $h=2^{-10}$ and measure the errors in the state $e_{k}^{\tau, y}$ and in the control $e_{k}^{\tau, u}=\left\|\bar{u}-\bar{u}_{k}\right\|_{L^{1}(0, T)}$ as the time step decreases. Error in the control can be computed exactly.

The experimental orders of convergence in space and time are defined as

$$
E O C_{i}^{h, y}=\frac{\log \left(e_{i+1}^{h, y}\right)-\log \left(e_{i}^{h, y}\right)}{\log \left(2^{-i-1}\right)-\log \left(2^{-i}\right)} \text { and } E O C_{k}^{\tau, *}=\frac{\log \left(e_{k+1}^{\tau, *}\right)-\log \left(e_{k}^{\tau, *}\right)}{\log \left(3^{-k-1}\right)-\log \left(3^{-k}\right)} .
$$

Since the error in space is much smaller than the error in time, we take $T=0.1$. For the optimization process, we choose $\nu_{0}=1 \mathrm{E}-4, \varepsilon=1 \mathrm{E}-14, \nu_{\min }=1 \mathrm{E}-8$. As a starting point we take $u_{0}=0, y_{0}=y_{\sigma}\left(u_{0}\right)$, and $\varphi_{0}=\varphi_{\sigma}\left(u_{0}\right)$. In all cases, the algorithm finishes in less than 8 iterations.

The results are shown in Table 1 . It can be noticed that the EOC in space is $O\left(h^{2}\right)$, while the EOC in time is $O(\tau)$. These results are quite in agreement, up to logarithmic terms, with the theoretical results given in Theorem 6.5.

7.2. Singular control. Define $\bar{\varphi}(x, t)=x(1-x)\left(1-\chi_{[0.25 T, 0.75 T]}(t)\right) \sin (4 \pi t / T)$ and

$$
\bar{u}(t)=\left\{\begin{array}{cl}
0 & \text { if } t<0.25 T \\
16(t-0.25 T)^{2}(T-t) / T^{3} & \text { if } 0.25 T \leq t \leq 0.75 T \\
1 & \text { if } t>0.75 T
\end{array}\right.
$$

We fix $T=1$. For the optimization process, we choose $\nu_{0}=1, \varepsilon=1 \mathrm{E}-14, \nu_{\min }=$ $1 \mathrm{E}-8$. We initialize the algorithm with $u_{0}=0, y_{0}=y_{\sigma}\left(u_{0}\right)$ and $\varphi_{0}=\varphi_{\sigma}\left(u_{0}\right)$. These 
TABLE 1

Error and EOC in space (left) and time (right). Bang-bang control. Example 7.1.

\begin{tabular}{c|c|c}
\hline \multicolumn{3}{|c}{$k=8$} \\
\hline$i$ & $e_{i}^{h, y}$ & $E O C_{i}^{h, y}$ \\
\hline 1 & $1.1 \mathrm{E}-2$ & - \\
2 & $2.7 \mathrm{E}-3$ & 2.0 \\
3 & $6.6 \mathrm{E}-4$ & 2.0 \\
4 & $1.7 \mathrm{E}-4$ & 2.0 \\
5 & $4.4 \mathrm{E}-5$ & 2.0 \\
6 & $1.3 \mathrm{E}-5$ & 1.8 \\
\hline
\end{tabular}

\begin{tabular}{c|c|c|c|c}
\hline \multicolumn{5}{c}{$i=10$} \\
\hline$k$ & $e_{k}^{\tau, y}$ & $E O C_{k}^{\tau, y}$ & $e_{k}^{\tau, u}$ & $E O C_{k}^{\tau, u}$ \\
\hline 1 & $5.0 \mathrm{E}-3$ & - & $1.7 \mathrm{E}-2$ & \\
2 & $1.7 \mathrm{E}-3$ & 1.0 & $5.6 \mathrm{E}-3$ & 1.0 \\
3 & $5.7 \mathrm{E}-4$ & 1.0 & $1.9 \mathrm{E}-3$ & 1.0 \\
4 & $1.9 \mathrm{E}-4$ & 1.0 & $6.2 \mathrm{E}-4$ & 1.0 \\
5 & $6.3 \mathrm{E}-5$ & 1.0 & $2.1 \mathrm{E}-4$ & 1.0 \\
6 & $2.1 \mathrm{E}-5$ & 1.0 & $6.9 \mathrm{E}-5$ & 1.0 \\
\hline
\end{tabular}
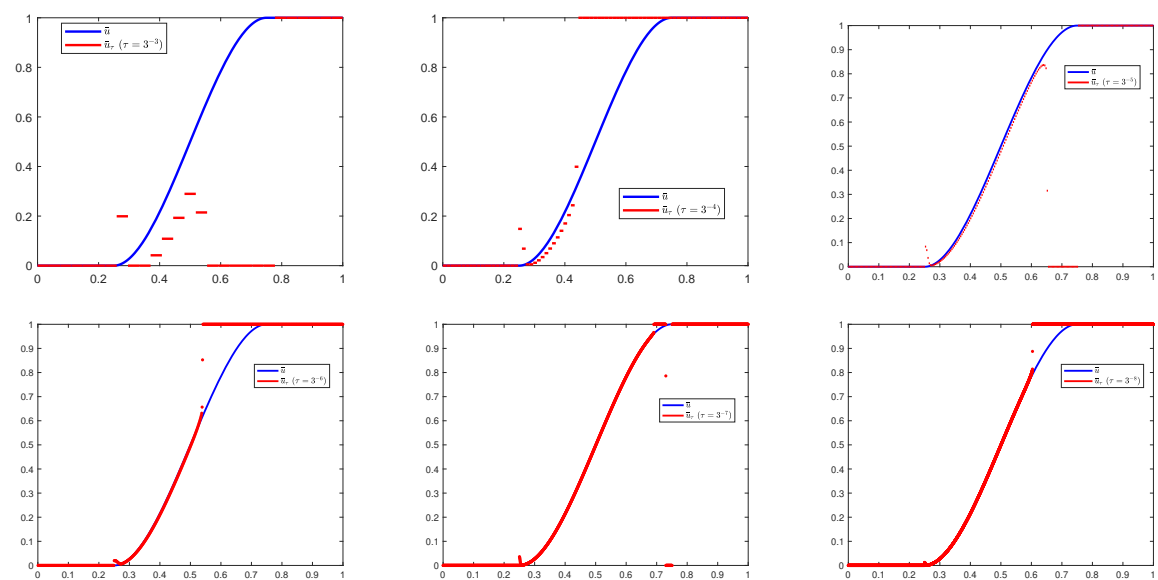

FIG. 1. Discrete optimal control for different time steps. Example 7.2.

TABLE 2

State error, and function values for problem with a singular arc. Example 7.2.

\begin{tabular}{c||c|c||c|c}
\hline$k$ & $e_{k}^{\tau, y}$ & $E O C_{k}^{\tau, y}$ & $J_{\sigma}\left(\bar{u}_{\sigma}\right)$ & $J_{\sigma}\left(\pi_{\tau} \bar{u}\right)$ \\
\hline 2 & $3.6 \mathrm{E}-2$ & & -0.04728096 & -0.04629675 \\
3 & $1.9 \mathrm{E}-2$ & 0.56 & -0.04639788 & -0.04619277 \\
4 & $1.0 \mathrm{E}-2$ & 0.60 & -0.04629958 & -0.04623007 \\
5 & $1.2 \mathrm{E}-2$ & -0.15 & -0.04623967 & -0.04622969 \\
6 & $3.6 \mathrm{E}-3$ & 1.1 & -0.04623509 & -0.04623015 \\
7 & $2.7 \mathrm{E}-3$ & 0.26 & -0.04623024 & -0.04623015 \\
8 & $1.4 \mathrm{E}-3$ & 0.60 & -0.04623044 & -0.04623016 \\
\hline & \multicolumn{3}{|c}{} \\
\hline
\end{tabular}

problems are much more difficult to solve: in all cases, the algorithm finished in about 40 iterations and discrete optimal controls are quite different from $\bar{u}$ (see Figure 1). Despite this, we have in all cases but one that $J_{\sigma}\left(\bar{u}_{\sigma}\right)<J_{\sigma}\left(\pi_{\tau} \bar{u}\right)$; see Table 2 .

Also, the order of convergence is harder to measure. We have only been able to perform experiment 2, i.e., we fix $h=2^{-10}$ and measure the error in the state for $\tau_{k}=3^{-k}, k=3, \ldots, 8$. The discretization errors are quite big and seemingly behave as $\sqrt{\tau}$ in time, which is in accordance with the result of Theorem 5.6; see Figure 2 and Table 2. The part of the error depending of $h$ is much smaller, and we have not been able to measure it.

Copyright $@$ by SIAM. Unauthorized reproduction of this article is prohibited. 


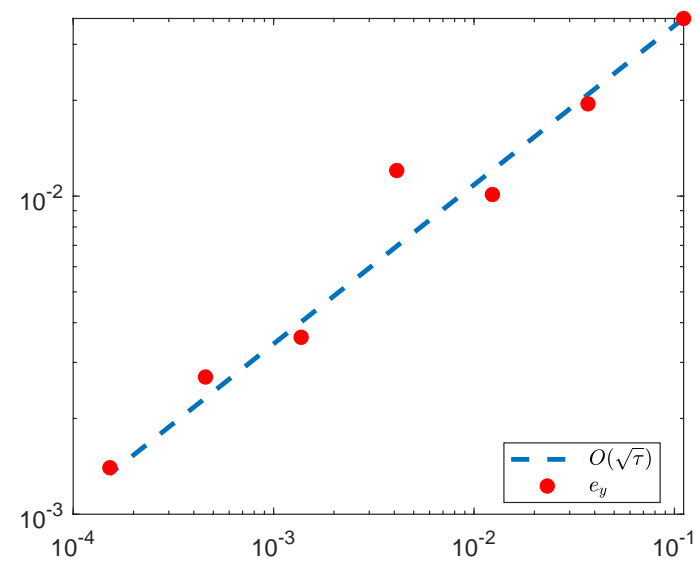

FIG. 2. Experimental and theoretical order of convergence for problem with a singular arc.

\section{REFERENCES}

[1] T. Bayen, F. Bonnans, And F. Silva, Characterization of local quadratic growth for strong minima in the optimal control of semi-linear elliptic equations, Trans. Amer. Math. Soc., 366 (2014), pp. 2063-2087.

[2] T. BAYEn AND F. Silva, Second order analysis for strong solutions in the optimal control of parabolic equations, SIAM J. Control Optim., 54 (2016), pp. 819-844.

[3] L. Bonifacius, K. PiePer, And B. Vexler, Error estimates for space-time discretization of parabolic time-optimal control problems with bang-bang controls, SIAM J. Control Optim., 57 (2019), pp. 1730-1756.

[4] F. Bonnans, Second-order analysis for control constrained optimal control problems of semilinear elliptic systems, Appl. Math. Optim., 38 (1998), pp. 303-325.

[5] F. Bonnans and A. Shapiro, Perturbation Analysis of Optimization Problems, SpringerVerlag, Berlin, 2000.

[6] S. C. Brenner and L. R. Scott, The Mathematical Theory of Finite Element Methods, 2nd ed., Texts Appl. Math. 15, Springer-Verlag, Berlin, 2002.

[7] E. CASAS, Pontryagin's principle for state-constrained boundary control problems of semilinear parabolic equations, SIAM J. Control Optim., 35 (1997), pp. 1297-1327.

[8] E. CASAS, Second order analysis for bang-bang control problems of PDEs, SIAM J. Control Optim., 50 (2012), pp. 2355-2372.

[9] E. CASAS AND K. ChRYSAFINOS, Error estimates for the approximation of the velocity tracking problem with bang-bang controls, ESAIM Control Optim. Calc. Var., 23 (2017), pp. 12671291.

[10] E. Casas and M. Mateos, Optimal Control of Partial Differential Equations, Springer-Verlag, Berlin, 2017, pp. 3-59.

[11] E. Casas, C. Ryll, And F. Tröltzsch, Second order and stability analysis for optimal sparse control of the FitzHugh-Nagumo equation, SIAM J. Control Optim., 53 (2015), pp. 21682202.

[12] E. Casas And F. TRÖltzsch, Second order analysis for optimal control problems: Improving results expected from abstract theory, SIAM J. Optim., 22 (2012), pp. 261-279.

[13] E. Casas And F. Tröltzsch, Second order optimality conditions and their role in PDE control, Jahresber. Dtsch. Math-Ver, 117 (2015), pp. 3-44.

[14] E. CASAS AND F. Tröltzsch, Second-order optimality conditions for weak and strong local solutions of parabolic optimal control problems, Vietnam J. Math., 44 (2016), pp. 181-202.

[15] E. Casas, D. Wachsmuth, and G. Wachsmuth, Second-order analysis and numerical approximation for bang-bang bilinear control problems, SIAM J. Control Optim., 56 (2018), pp. 4203-4227.

[16] E. Casas, D. Wachsmuth, And G. Wachsmuth, Sufficient second-order conditions for bangbang control problems, SIAM J. Control Optim., 55 (2017), pp. 3066-3090.

[17] N. V. Daniels And M. Hinze, Variational discretization of a control-constrained parabolic bang-bang optimal control problem, J. Comput. Math., 37 (2019), pp. 361-387.

Copyright $@$ by SIAM. Unauthorized reproduction of this article is prohibited. 
[18] K. Deckelnick And M. Hinze, A note on the approximation of elliptic control problems with bang-bang controls, Comput. Optim. Appl., 51 (2012), pp. 931-939.

[19] J. DunN, On second order sufficient optimality conditions for structured nonlinear programs in infinite-dimensional function spaces, in Mathematical Programming with Data Perturbations, A. Fiacco, ed., Marcel Dekker, New York, 1998, pp. 83-107.

[20] O. A. Ladyženskaja, V. A. Solonnikov, and N. N. URAL'Ceva, Linear and Quasilinear Equations of Parabolic Type, Transl. Math. Monogr. 23, American Mathematical Society, Providence, RI, 1968.

[21] H. Maurer And J. Zowe, First and second order necessary and sufficient optimality conditions for infinite-dimensional programming problems, Math. Program., 16 (1979), pp. 98-110.

[22] D. Meidner and B. Vexler, Optimal error estimates for fully discrete Galerkin approximations of semilinear parabolic equations, ESAIM Math. Model. Numer. Anal., 52 (2018), pp. 2307-2325.

[23] F. PÖRNER AND D. WAChSMUth, Tikhonov regularization of optimal control problems governed by semi-linear partial differential equations, Math. Control Relat. Fields, 8 (2018), pp. 315335.

[24] N. Qui And D. WachSmuth, Stability for bang-bang control problems of partial differential equations, Optimization, 67 (2018), pp. 2157-2177.

Copyright (c) by SIAM. Unauthorized reproduction of this article is prohibited. 